\title{
Diffuse interstellar bands in the $\mathrm{H}$ II region M17
}

\section{Insights into their relation with the total-to-selective visual extinction $\boldsymbol{R}_{V}^{\star}$}

\author{
M. C. Ramírez-Tannus ${ }^{1}$, N. L. J. Cox $^{2}$, L. Kaper ${ }^{1}$, and A. de Koter ${ }^{1,3}$ \\ 1 Anton Pannekoek Institute for Astronomy, University of Amsterdam, Science Park 904, 1098 XH Amsterdam, The Netherlands \\ e-mail: m.c.ramireztannus@uva.nl \\ 2 ACRI-ST, 260 Route du Pin Montard, Sophia Antipolis, France \\ ${ }^{3}$ Institute of Astronomy, KU Leuven, Celestijnenlaan 200D, 3001 Leuven, Belgium
}

Received 2 May 2018 / Accepted 30 August 2018

\begin{abstract}
Context. Diffuse interstellar bands (DIBs) are broad absorption features measured in sightlines probing the diffuse interstellar medium. Although large carbon-bearing molecules have been proposed as the carriers producing DIBs, their identity remains unknown. DIBs make an important contribution to the extinction curve; the sightline to the young massive star-forming region M17 shows anomalous extinction in the sense that the total-to-selective extinction parameter $\left(R_{V}\right)$ differs significantly from the average Galactic value and may reach values $R_{V}>4$. Anomalous DIBs have been reported in the sightline towards Herschel $36\left(R_{V}=5.5\right)$, in the massive star-forming region M8. Higher values of $R_{V}$ have been associated with a relatively higher fraction of large dust grains in the line of sight.

Aims. Given the high $R_{V}$ values, we investigate whether the DIBs in sightlines towards young OB stars in M17 show a peculiar behaviour.

Methods. We measure the properties of the most prominent DIBs in M17 and study these as a function of $E(B-V)$ and $R_{V}$. We also analyse the gaseous and dust components contributing to the interstellar extinction.

Results. The DIB strengths in M17 concur with the observed relations between DIB equivalent width and reddening $E(B-V)$ in Galactic sightlines. For several DIBs we discover a linear relation between the normalised DIB strength EW/ $/ A_{V}$ and $R_{V}^{-1}$. These trends suggest two groups of DIBs: (i) a group of ten moderately strong DIBs that show a sensitivity to changes in $R_{V}$ that is modest and proportional to DIB strength, and (ii) a group of four very strong DIBs that react sensitively and to a similar degree to changes in $R_{V}$, but in a way that does not appear to depend on DIB strength.

Conclusions. DIB behaviour as a function of reddening is not peculiar in sightlines to M17. Also, we do not detect anomalous DIB profiles like those seen in Herschel 36. DIBs are stronger, per unit visual extinction, in sightlines characterised by a lower value of $R_{V}$, i.e. those sightlines that contain a relatively large fraction of small dust particles. New relations between extinction normalised DIB strengths, EW/A $A_{V}$, and $R_{V}$ support the idea that DIB carriers and interstellar dust are intimately connected. Furthermore, given the distinct behaviour of two groups of DIBs, different types of carriers do not necessarily relate to the dust grains in a similar way.
\end{abstract}

Key words. dust, extinction - HII regions - ISM: lines and bands

\section{Introduction}

The nature of the carrier(s) of diffuse interstellar bands (DIBs) is one of the oldest mysteries in stellar spectroscopy. Over 400 DIBs have been observed in the optical wavelength range (e.g. Hobbs et al. 2009) and about a dozen DIBs have been detected in the near-infrared (NIR; Geballe et al. 2011; Cox et al. 2014; Hamano et al. 2015). DIBs are thought to be large carbonbearing molecules and may represent the largest reservoir of organic material in the Universe (Snow 2014). Laboratory experiments simulating interstellar conditions recently proposed $\mathrm{C}_{60}^{+}$ as the carrier of the $\lambda 9577$ and $\lambda 9632$ DIBs (Campbell et al. 2015), but the vast majority of the DIBs remains unidentified. For a recent overview of DIBs, see Cami \& Cox (2014).

Diffuse interstellar bands measured in sightlines towards massive star-forming regions are reported to behave differently to the average Galactic sightline. Hanson et al. (1997) noted that the DIBs observed in the direction of M17, over the extinction

\footnotetext{
* Table 3 is also available at the CDS via anonymous ftp to cdsarc.u-strasbg.fr (130.79.128.5) or via http://cdsarc.ustrasbg.fr/viz-bin/qcat?J/A+A/620/A52
}

range of $A_{V}=3-10$, show little change in spectral shape nor a significant increase in strength. They suggested that either the DIB features are already saturated at a low value of the visual extinction $A_{V}$ or that the interstellar material local to M17, where the increased extinction is being traced, does not contain the DIB carriers. Dahlstrom et al. (2013) and Oka et al. (2013) detected anomalously broad DIBs at $\lambda 5780.5,5797.1$, and 6613.6 in the sightline to Herschel 36, an $\mathrm{O}$ star multiple system associated with the Hourglass nebula in the giant $\mathrm{H}$ II region M8 (Lagoon Nebula). The DIBs show an excess of absorption in the red wing of the profile; excited lines of $\mathrm{CH}$ and $\mathrm{CH}^{+}$are detected as well. Oka et al. (2013) interpret this observation as being caused by infrared pumping of rotational levels of relatively small molecules.

Sightlines towards massive star-forming regions (Orion Trapezium region, Carina, M8) often show a high value of the total-to-selective extinction parameter $R_{V}$ (see e.g. Cardelli et al. 1989; Fitzpatrick \& Massa 2007). The common interpretation is that the value of $R_{V}$ characterises the dust particle size distribution. Sightlines that include many small dust particles display an extinction curve with a low value of $R_{V}$ and produce stronger 
interstellar absorption at short (UV) wavelengths, and vice versa. Most Galactic sightlines have $R_{V} \sim 3.1 \pm 0.3$ (Fitzpatrick \& Massa 2007) and we refer to extinction curves with higher or lower values of $R_{V}$ as being anomalous.

The strongest feature in the extinction curve is the $2175 \AA$ bump; although the carrier of this feature also remains unidentified, it is generally attributed to carbonaceous particles, either in the form of graphite, a mixture of hydrogenated amorphous carbon grains and polycyclic aromatic hydrocarbons (PAHs), or various aromatic forms of carbon (e.g. Mathis et al. 1977; Mennella et al. 1998; Mishra \& Li 2015). It is believed that the presence and strength of the bump depends on the metallicity of the environment as it appears slightly weaker in the LMC extinction curves, and it is essentially absent in the SMC extinction curve (Gordon \& Clayton 1998; Misselt et al. 1999). Some sightlines towards the LMC (specifically the LMC 2 supershell near 30 Dor) and the SMC sightlines have low $R_{V}$ values ( 2.7; Gordon et al. 2003). Remarkably, the one SMC line of sight that exhibits normal strength DIBs (and $\mathrm{CH}$ ) shows Galactictype dust extinction and includes the $2175 \AA$ extinction feature (Ehrenfreund et al. 2002; Welty et al. 2006; Cox et al. 2007). Furthermore, Galactic sightlines that are SMC-like show very weak DIBs per unit reddening (Snow et al. 2002; Cox et al. 2007).

If the carriers of the DIBs and those of the $2175 \AA$ bump are produced from the same initial dust through the same physical process, or if the DIB carriers originate from the fragmentation of the $2175 \AA$ bump carriers, we would expect them to be related. More recently, Xiang et al. (2011) studied the relation between DIBs and the $2175 \AA$ bump in detail. They collected $2175 \AA$ bump and DIB strength measurements from the literature towards 84 interstellar sightlines for eight DIBs, and found no significant correlation between the two. They explain the lack of correlation by hypothesising that DIB carriers correspond to the smallest, free-flying PAH molecules and ions, while the $2175 \AA$ carriers correspond to the larger or clustered PAHs.

In Ramírez-Tannus et al. (2017; hereafter Paper I), we studied a sample of young massive (pre-)main-sequence stars in the giant $\mathrm{H}$ II region M17 located at a distance of $1.98_{-0.12}^{+0.14} \mathrm{kpc}$ (Xu et al. 2011). We obtained optical to NIR VLT/X-shooter spectra and derived the extinction parameters by modelling the spectral energy distribution. The sightlines are characterised by anomalous extinction $\left(R_{V}\right.$ in the range 3.3-4.6 and $A_{V}$ between 5 and $14 \mathrm{mag}$ ). M17 is one of the most luminous starforming regions in the Galaxy with a luminosity if $3.6 \times 10^{6} \mathrm{~L}_{\odot}$ (Prisinzano et al. 2007). It contains about $16 \mathrm{O}$ stars and more than 100 B stars; its age is $\leq 1$ Myr (Hanson et al. 1997; Broos et al. 2007; Hoffmeister et al. 2008; Povich et al. 2009; Paper I).

In this paper, we present a detailed analysis of the DIBs in eight sightlines to M17, taking into account the anomalous extinction observed in this region $\left(R_{V}>3.1\right)$. An earlier work hinted at the peculiar behaviour of the DIBs in these sightlines (Hanson et al. 1997) and we investigate whether the DIB properties are somehow related to the extinction caused by dust. Such a relation may shed new light on the physical and chemical nature of the DIB carriers.

The paper is organised as follows. In the next section we briefly describe the data set and reduction procedure, and in Sect. 3, we characterise the interstellar extinction in the direction of M17 of both the gaseous and the dust components. In Sect. 4, the DIB properties are presented. Subsequently, we compare the DIB properties to those observed in other Galactic sightlines (Sect. 5). In Sect. 6, we address the observed dependence of

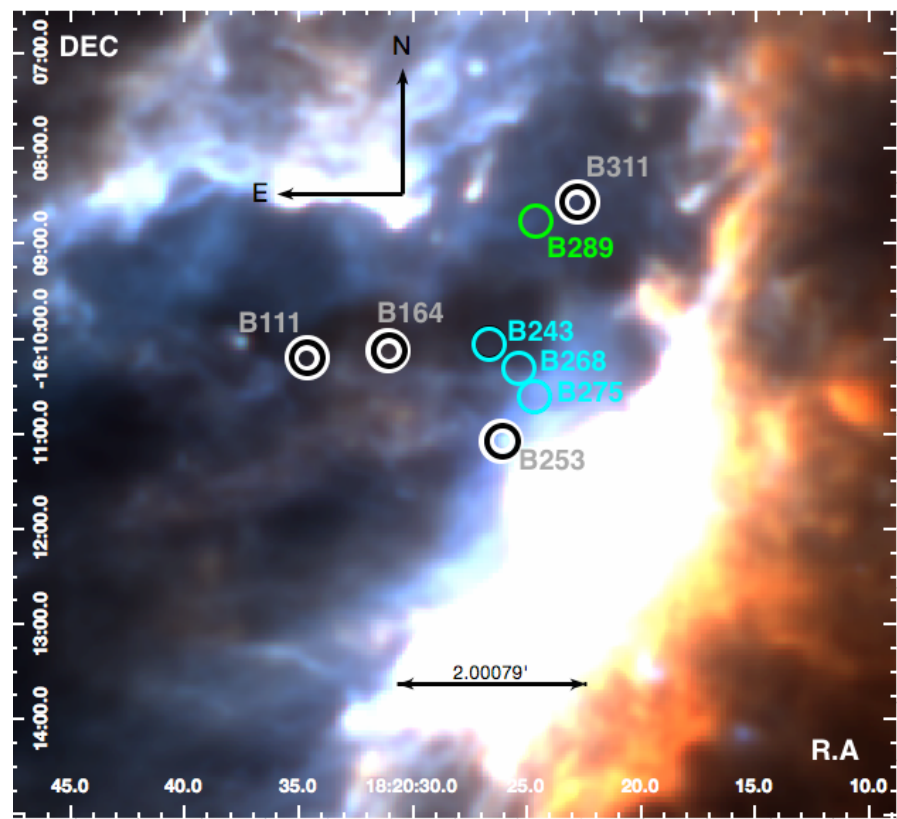

Fig. 1. Far-infrared colour composite image of M17 based on Herschel PACS data. Blue: $70 \mu \mathrm{m}$, green: $100 \mu \mathrm{m}$, and red: $160 \mu \mathrm{m}$. The sources with circumstellar disks are shown in blue, those with IR excess longwards of $2.5 \mu \mathrm{m}$ in green, and the "naked" OB stars in black. We do not observe an obvious trend in extinction properties with the location in the region.

the DIBs in M17 on the value of $R_{V}$. In Sect. 7, we discuss the results in the context of the anomalous extinction and the properties of the interstellar dust. Finally, in Sect. 8 we summarise our conclusions.

\section{X-shooter observations}

We have obtained VLT/X-shooter (300-2500 nm; Vernet et al. 2011) spectra of 11 (pre-)main sequence (PMS) stars ranging in mass from 6 to $20 M_{\odot}$. We selected this sample from the list of candidate massive PMS stars reported by Hanson et al. (1997) and we determined their stellar and extinction properties, which are presented in Paper I. The location of the observed targets in M17 is shown in Fig. 1. With the exception of Fig. 10, throughout this paper we have colour-coded the M17 sightlines according to the properties of the stellar spectral energy distributions. The blue circles represent the sightlines towards objects with circumstellar disks (gas+dust disks), the green triangles towards stars with IR excess longwards of $2.5 \mu \mathrm{m}$ (dusty disks), and the black squares towards OB stars without detected circumstellar material. We reduced the spectra using the X-shooter pipeline (Modigliani et al. 2010) version 2.7.1 running under the ESO Reflex environment (Freudling et al. 2013) version 2.8.4. The flux calibration was obtained using spectrophotometric standards from the ESO database. We then scaled the NIR flux to match the absolutely calibrated VIS spectrum. The telluric correction was performed using the software tool molecfit v1.2.0 ${ }^{1}$ (Smette et al. 2015; Kausch et al. 2015). The data reduction and analysis are described in detail in Paper I.

\footnotetext{
http://www . eso.org/sci/software/pipelines/skytools/ molecfit
} 
Table 1. Extinction properties measured in the sightlines towards M17.

\begin{tabular}{cccccccccccc}
\hline \hline Identifier & Sp.type & $\begin{array}{c}B \\
\text { mag }\end{array}$ & $\begin{array}{c}V \\
\mathrm{mag}\end{array}$ & $\begin{array}{c}K^{a} \\
\mathrm{mag}\end{array}$ & $\begin{array}{c}R_{V} \\
\text { SED }\end{array}$ & $\begin{array}{c}R_{V} \\
\text { Fitzp. }\end{array}$ & $\begin{array}{c}A_{V} \\
\text { SED (mag) }\end{array}$ & $\begin{array}{c}A_{V} \\
V-V_{0}\end{array}$ & $\begin{array}{c}E(B-V) \\
\text { SED }\end{array}$ & $\begin{array}{c}E(B-V) \\
(B-V)-\left(B-V_{0}\right.\end{array}$ & $\begin{array}{c}A_{K} \\
\text { mag }\end{array}$ \\
\hline B111 & O4.5 V & $12.24^{b}$ & $11.21^{b}$ & 7.475 & $3.7_{-0.3}^{+0.4}$ & 3.86 & $5.4_{-0.3}^{+0.4}$ & 5.17 & $1.47 \pm 0.13$ & 1.35 & 0.50 \\
B164 & O6 Vz & $16.85^{c}$ & $15.41^{c}$ & 8.758 & $3.8_{-0.3}^{+0.3}$ & 4.87 & $8.3_{-0.5}^{+0.4}$ & 9.03 & $2.2 \pm 0.10$ & 1.76 & 1.44 \\
B215 & B0-B1 V & - & $16.10^{d}$ & 10.00 & $4.1_{-0.2}^{+0.2}$ & - & $7.6_{-0.3}^{+0.3}$ & 8.17 & $1.85 \pm 0.06$ & - & 1.15 \\
B243 & B8 V & $19.10^{c}$ & $17.80^{c}$ & 9.544 & $4.7_{-0.8}^{\uparrow}$ & 4.64 & $8.5_{-1.0}^{\uparrow}$ & 6.52 & $1.82 \pm 0.22$ & 1.41 & 0.72 \\
B253 & B3-B5 III & $17.11^{c}$ & $15.74^{c}$ & 10.31 & $4.1_{-0.2}^{+0.1}$ & 4.29 & $6.5_{-0.2}^{+0.1}$ & 6.22 & $1.59 \pm 0.06$ & 1.52 & 0.41 \\
B268 & B9-A0 & $18.40^{c}$ & $17.10^{c}$ & 9.494 & $4.6_{-0.8}^{\dagger}$ & 3.47 & $8.1_{-1.0}^{\uparrow}$ & 4.71 & $1.75 \pm 0.25$ & 1.34 & 0.52 \\
B275 & B7 III & $17.02^{c}$ & $15.55^{c}$ & 7.947 & $3.8_{-0.8}^{+0.7}$ & 3.35 & $6.7_{-1.0}^{+0.8}$ & 5.44 & $1.77 \pm 0.26$ & 1.59 & 0.61 \\
B289 & O9.7 V & $16.97^{c}$ & $15.55^{c}$ & 9.178 & $3.7_{-0.5}^{+0.4}$ & 4.80 & $8.3_{-0.8}^{+0.6}$ & 8.13 & $2.23 \pm 0.17$ & 1.73 & 0.78 \\
B311 & O8.5 Vz & $14.99^{c}$ & $13.69^{c}$ & 8.884 & $3.3_{-0.3}^{+0.3}$ & 4.00 & $6.1_{-0.4}^{+0.4}$ & 6.51 & $1.85 \pm 0.11$ & 1.62 & 0.71 \\
B331 & late-B & - & $20.10^{c}$ & 8.946 & $4.6_{-0.5}^{+0.5}$ & - & $13.3_{-0.9}^{+0.9}$ & - & $2.89 \pm 0.13$ & - & - \\
B337 & late-B & - & - & 9.343 & $3.7_{-0.5}^{+0.4}$ & - & $8.3_{-0.8}^{+0.6}$ & - & $3.66 \pm 0.25$ & - & - \\
\hline
\end{tabular}

Notes. Column 1 lists the star's identifier, Col. 2 the spectral type as reported in Paper I. Columns 3-5 show the $B, V$, and $K$-band magnitudes, respectively. Columns 6,8 , and 10 list the extinction parameters $\left(R_{V}, A_{V}\right.$, and $\left.E(B-V)=A_{V} / R_{V}\right)$ derived by fitting the spectral energy distribution to Kurucz models. We list the $R_{V}$ and $A_{K}$ values derived using Eq. (1) (see Sect. 3 for more information on objects with circumstellar disks) in Cols. 7 and 12. Column 9 lists the $V$-band excess $A_{V}$, and Col. 11 shows the colour excess $(B-V)-(B-V)_{0}$, where the intrinsic colour $(B-V)_{0}$ is taken from Pecaut \& Mamajek (2013) and Wegner (2000, 2014). ${ }^{(a)}$ 2MASS catalogue (Cutri et al. 2003). ${ }^{(b)}$ AAVSO Photometric all sky survey (APASS) catalog: https: //www . aavso. org/apass ${ }^{(c)}$ Chini et al. (1980). ${ }^{(d)}$ Hoffmeister et al. (2008).

We calculated the stellar properties of the objects with visible photospheres by fitting FASTWIND models (Puls et al. 2005; Rivero González et al. 2012) and obtained the total extinction in the $V$-band, $A_{V}$, and the visual-to-selective extinction ratio, $R_{V}$, by fitting the flux calibrated $\mathrm{X}$-shooter spectra to Castelli \& Kurucz (2004) models. The spectral type and extinction properties presented in Paper I are listed in Table 1. In this paper we compare the results obtained in Paper I where we use the Cardelli et al. (1989) extinction law to deredden the flux calibrated spectra with results obtained by using the Fitzpatrick \& Massa (2007) parameterisation of the extinction curve. We also compare the extinction parameters obtained by SED fitting with that derived from the colour excess $E(B-V)$ (see Sect. 3). We also list the $R_{V}$ value obtained from Eq. (1); for objects with a NIR excess (i.e. a circumstellar disk: B243, B268, and B275) we iterated this equation starting with $A_{K}=0$. In this way we obtain an estimate of the $K$-band extinction $A_{K}$, and thus a measure of the NIR excess (Sect. 3).

\section{Extinction towards M17}

By dereddening the SEDs of a dozen OB stars Hanson et al. (1997) detected a significant spread in both visual extinction $A_{V}$ (3-15 mag) and in total-to-selective extinction $R_{V}(2.8-5.5)$. They point out that the nebulosity disappears at the centre of the region and that the highest extinction is measured at the edges of this void. They also note that the DIB strengths (4430 and $4502 \AA$ ) do not show a significant increase even though the visual extinction towards these objects varies from 3-10 mag. Earlier studies indicated that $R_{V}=4.9$ (Chini \& Kruegel 1983) based on NIR photometry (but without knowledge of the spectral types), and $R_{V}=3.3$ using the maximum polarisation relation for $R_{V}$ (Schulz et al. 1981). Hoffmeister et al. (2008) concluded that the foreground extinction, with $A_{V} \sim 2$ mag and $R_{V}=3.1$, differs from the extinction local to M17 for which they measure $R_{V}=3.9 \pm 0.2$.

\subsection{Dust extinction}

In Paper I we constructed the spectral energy distribution (SED) of the stars by dereddening the X-shooter spectrum complemented with available photometric data. In order to deredden the spectrum we used the Cardelli et al. (1989) extinction law. We then constrained $A_{V}$ and $R_{V}$ towards the objects in M17 by fitting the slope of their SED in the photospheric domain $\left(400-820 \mathrm{~nm}\right.$ ) to Castelli \& Kurucz models ${ }^{2}$ (Kurucz 1993; Castelli \& Kurucz 2004) corresponding to the spectral type given in Table 1.

The obtained values for $R_{V}$ range from 3.3 to 4.7 and $A_{V}$ varies from $\sim 6$ to $\sim 15 \mathrm{mag}$. We do not find a significant correlation of the extinction properties with the position of the stars in the star-forming region. Cardelli et al. (1989) mention that their parameterisation of the extinction law becomes inaccurate when $R_{V}$ is large. As an example they refer to the sightline to Herschel 36.

As we know the spectral type of the targets, we can compare the value of the colour excess $E(B-V) \equiv A_{V} / R_{V}$ measured by fitting the SED with the value obtained directly by comparing the observed magnitudes to the intrinsic ones $(E(B-V)=$ $\left.(B-V)-(B-V)_{0}\right)$. We adopt an error of $0.1 \mathrm{mag}$ in $E(B-V)$ in order to account for the uncertainty in the spectral classification and the photometric errors. For the main sequence stars we took the value of $B_{0}$ and $V_{0}$ from Pecaut \& Mamajek (2013). For the giants we used the calibration in Wegner $(2000,2014)$. The comparison is shown in the left panel of Fig. 2. The value of $E(B-V)$ derived from the SED fitting method is systematically higher than the one obtained directly from the observed and the intrinsic colours. The $E(B-V)$ values obtained with the SED fitting method and the Fitzpatrick (1999) extinction law are closer to those obtained directly from the colours and we do not observe a systematic trend. Apparently, we underestimate the value of 2 Table 2 in http://www.stsci.edu/hst/observatory/crds/
castelli_kurucz_atlas.html 

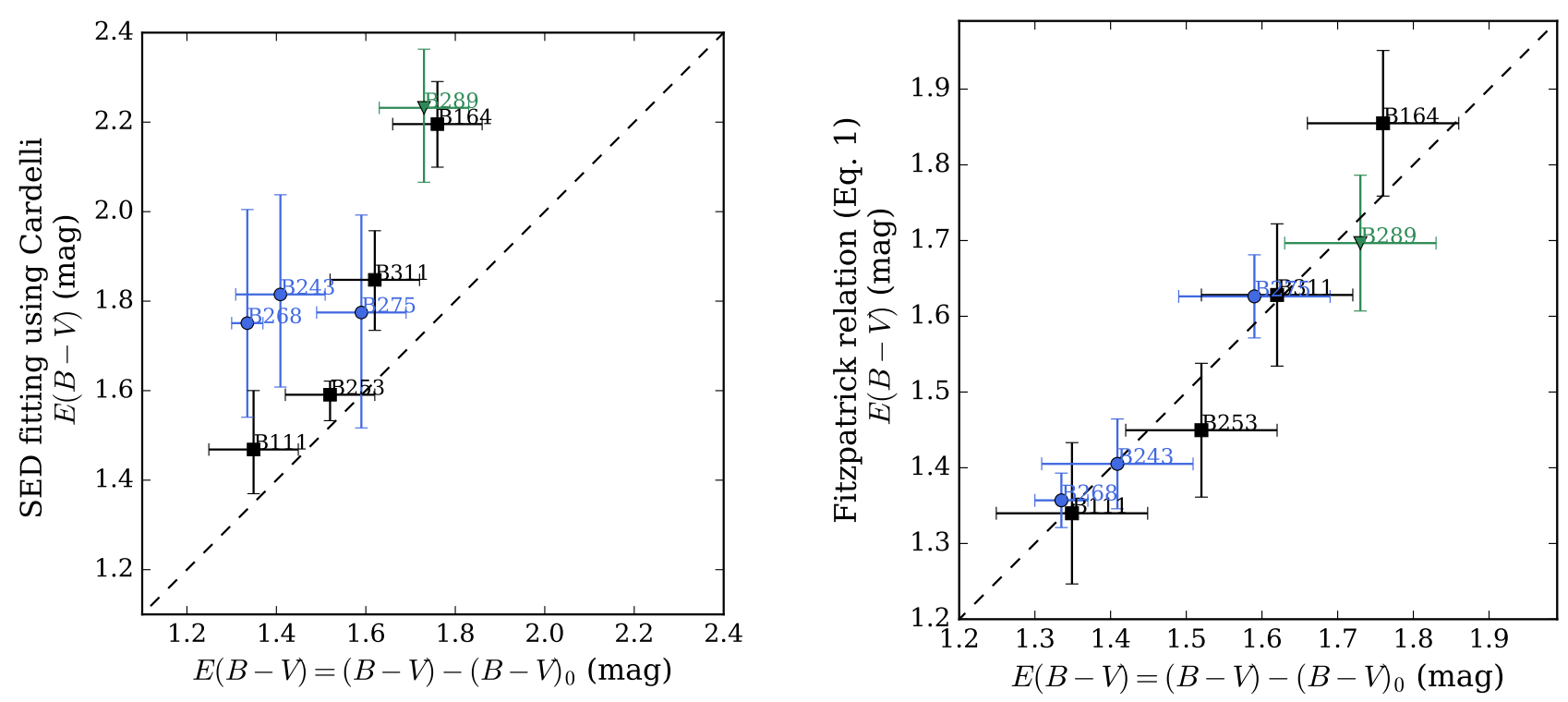

Fig. 2. Left panel: colour excess $E(B-V)$ derived from SED fitting $\left(A_{V} / R_{V}\right.$, Paper I) vs. the value obtained using the observed and the intrinsic colour $(B-V)_{0}$; the latter is based on the spectral type reported in Paper I. Right panel: colour excess obtained from $A_{V} / R_{V}$, where $R_{V}$ is estimated using Eq. (1) in Fitzpatrick \& Massa (2007), vs. the value obtained by using the observed and the intrinsic colour, as in the left panel. Now the discrepancy between these two values has been removed, demonstrating that the Cardelli et al. (1989) parameterisation underestimates the value of $R_{V}$ in these sightlines. The blue circles represent the sightlines towards objects with circumstellar disks, the green triangles towards stars with dusty disks, and the black squares towards OB stars.

$R_{V}$ with the SED fitting method and the Cardelli et al. (1989) extinction law.

In order to investigate this further, we calculated $R_{V}$ using the relation reported in Fitzpatrick \& Massa (2007):

$R_{V}=-0.26+1.19 \frac{E(V-K)}{E(B-V)}=-0.26+1.19 \frac{A_{V}-A_{K}}{A_{B}-A_{V}}$.

Given that this relation uses the $K$-band magnitude, it implicitly assumes that the target's SED does not include an IR excess or, alternatively, that this is corrected for. In the case of the three objects with circumstellar disks (blue dots in the plots), we calculated $R_{V}$ by iterating Eq. (1). With $A_{V}=V-V_{0}$ and $E(B-V)=(B-V)-(B-V)_{0}$, we started assigning a value of $A_{K}=0$ and calculated $R_{V}$ from this equation; we then used Fitzpatrick's extinction law to calculate $A_{K}$ with the obtained $R_{V}$ and repeated this process until the values for both $R_{V}$ and $A_{K}$ converged. We arrived at $R_{V}=4.6,3.3$, and 3.1 for B243, B268, and B275, respectively ${ }^{3}$. The comparison between the colour excess calculated with Fitzpatrick's extinction law and the excess based on the observed and intrinsic colour is shown in the right panel of Fig. 2. From the figure it is clear that the value obtained using these two methods agree within the errors. In the case of objects with near-IR excess, we note that these two methods are not completely independent because we used the value based on the observed and intrinsic colour as initial guess to iterate Eq. (1).

We conclude that with the method used in Paper I, where the Cardelli et al. (1989) extinction law is used to deredden the SEDs for sightlines with high $R_{V}$, we underestimate the value of this parameter. Furthermore, we observe that the values for $A_{V}$ obtained with the three methods agree with each other. This

\footnotetext{
3 With this accurate measurement of the extinction properties we calculate $K$-band excesses of 2-3 magnitudes for B243, B268, and B275. In a forthcoming paper (Poorta et al., in prep.) we investigate the physical properties of the circumstellar disks that cause the measured $K$-band excess.
}

means that we did not make a significant error when calculating the luminosity of the objects in Paper I.

We do not find a systematic difference between the values obtained for objects with circumstellar gaseous disks (blue dots), only dusty disks (green triangle), and naked OB stars (black squares).

In the remainder of this paper we will adopt the values of $A_{V}=V-V_{0}, E(B-V)=(B-V)-(B-V)_{0}$, and $R_{V}$ derived from Eq. (1).

\subsection{Gaseous component of the extinction}

The CaII, Na I, and KI interstellar lines are shown in Fig. 3; we have overplotted the DIB at $5780 \AA$. The velocities have been corrected into the barycentric rest frame. In Table 2, we list the local standard rest (LSR) frame velocity measured for each of the lines. These radial velocities are consistent with interstellar absorption at distances of 1.3-2.2 kpc based on Galactic rotation models by Reid et al. (2009). We observe hardly any difference from one sightline to another. The atomic lines are mostly unresolved at the moderate $\mathrm{X}$-shooter spectral resolution of $R \sim 11000$. Although inconclusive regarding the exact distribution of gas in these lines of sight, these data indicate that the main gaseous absorption features are associated with M17 at $\sim 2 \mathrm{kpc}$.

From 3D dust extinction maps (Drimmel et al. 2003) we find that the predicted visual extinction up to $1.8 \mathrm{kpc}$ in the direction of M17 (i.e. in the foreground) corresponds to roughly $2 \mathrm{mag}$ (or $E(B-V)=0.65 \mathrm{mag}$ for a canonical value $R_{V}=$ 3.1), which is consistent with Hoffmeister et al. (2008). From the $E(B-V)$ versus distance towards M17 plot obtained via the online tool STILISM ${ }^{4}$ by Capitanio et al. (2017) we obtain a value of $E(B-V)=0.78 \mathrm{mag}$ at the distance M17, which is in line with our result. This plot shows that there are diffuse clouds

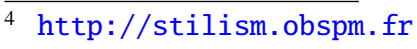




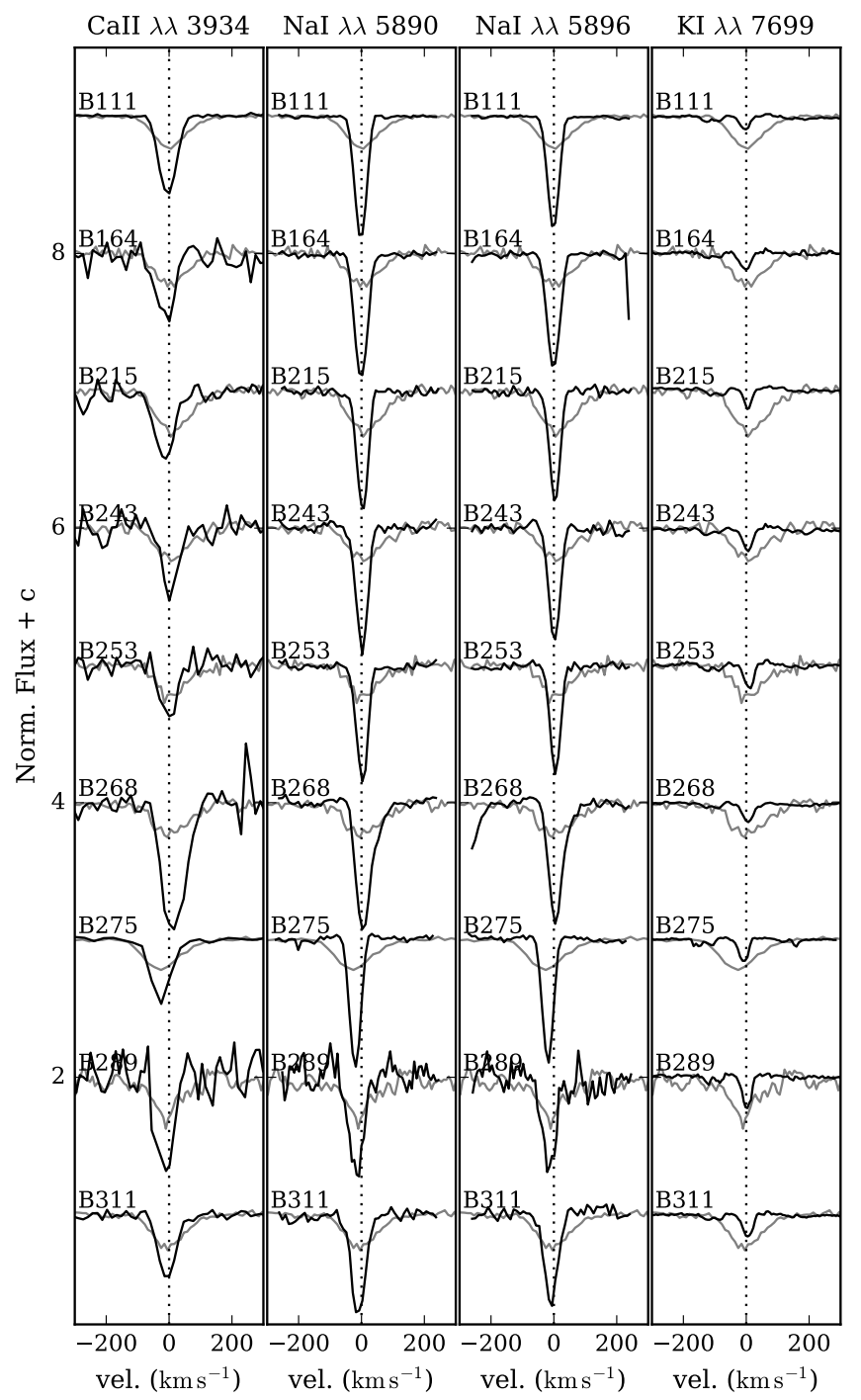

Fig. 3. Velocity profiles for the Ca II, Na I, and K I lines (black). We have overplotted the DIB at $5780 \AA$ A for reference (grey). The velocities are in the barycentric rest frame. The interstellar (gaseous) absorption profile is very similar from one sightline to another, and is likely due to a single cloud.

in the foreground to the local gas and dust associated with the M17 region.

\section{Diffuse interstellar bands}

In this section, we provide an overview of the DIB properties measured in the sightlines to the young $\mathrm{OB}$ stars in the star-forming region M17. We analysed the nine strongest DIBs present in the spectra obtained by the UVB and VIS arms of X-shooter (at 4430, 5780, 5797, 6196, 6284, 6379, 6614, 7224, and $8620 \AA$ ). We also included the NIR DIBs at 11797 and 13176, first reported by Joblin et al. (1990), and the one at 15268 (Geballe et al. 2011; Cox et al. 2014). The DIB equivalent width (EW) and full width at half maximum (FWHM) are listed in Table 3. The first column corresponds to the central wavelength of the DIBs; the top values show the FWHM in nm, and the

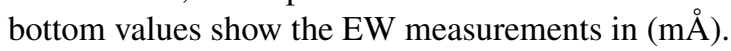

We also detect the 9577 and 9632 DIBs whose carrier has recently been identified in the laboratory to be the $\mathrm{C}_{60}^{+}$molecule
Table 2. Local standard rest frame (LSR) radial velocities obtained from a Gaussian fit to the Ca II, Na I, and K I interstellar lines in each line of sight.

\begin{tabular}{|c|c|c|c|c|}
\hline $\begin{array}{c}\text { Identifier } \\
\lambda \lambda\end{array}$ & $\begin{array}{c}\mathrm{Ca} \mathrm{II} \\
3933.66 \\
\mathrm{~km} \mathrm{~s}^{-1}\end{array}$ & $\begin{array}{c}\mathrm{Na} \mathrm{I} \\
5889.95 \\
\mathrm{~km} \mathrm{~s}^{-1}\end{array}$ & $\begin{array}{c}\mathrm{Na} \mathrm{I} \\
5895.92 \\
\mathrm{~km} \mathrm{~s}^{-1}\end{array}$ & $\begin{array}{c}\mathrm{K} \mathrm{I} \\
7698.974 \\
\mathrm{~km} \mathrm{~s}^{-1} \\
\end{array}$ \\
\hline B 111 & $9.3 \pm 0.4$ & $11.3 \pm 0.2$ & $12.1 \pm 0.2$ & - \\
\hline B 164 & $0.8 \pm 3.5$ & $12.6 \pm 0.3$ & $12.7 \pm 1.3$ & $10.4 \pm 1.3$ \\
\hline B215 & $-1.2 \pm 2.3$ & $16.8 \pm 0.3$ & $16.7 \pm 0.3$ & $18.9 \pm 1.0$ \\
\hline B243 & $16.6 \pm 13.5$ & $15.4 \pm 0.4$ & $15.7 \pm 0.5$ & $18.2 \pm 1.4$ \\
\hline B253 & $10.7 \pm 6.1$ & $16.2 \pm 0.4$ & $18.6 \pm 0.3$ & $22.4 \pm 1.1$ \\
\hline B268 & $28.8 \pm 2.2$ & $21.7 \pm 0.8$ & $20.4 \pm 1.4$ & $19.6 \pm 1.5$ \\
\hline B275 & $-13.2 \pm 1.0$ & $-6.1 \pm 0.4$ & $-5.1 \pm 0.3$ & $5.1 \pm 1.1$ \\
\hline B289 & $-9.1 \pm 5.2$ & $-11.3 \pm 1.6$ & $-9.9 \pm 1.7$ & - \\
\hline B311 & $7.3 \pm 1.1$ & $4.1 \pm 0.7$ & $4.6 \pm 0.8$ & - \\
\hline
\end{tabular}

(Campbell et al. 2015). The FWHM and EW of these two DIBs are displayed in rows 10 and 11 of Table 3.

We searched for the presence of $\mathrm{CH}^{+}$and $\mathrm{CH}$ at 4232 , $4300 \AA$, but given the signal-to-noise ratio of our data at such blue wavelengths we were not able to detect these molecules in the X-shooter spectra.

\subsection{DIB strengths and widths}

The EW was measured by integrating the flux of each DIB within the boundaries shown in Appendix A. The error on the EW was calculated following the method described in Vos et al. (2011): $\sigma_{\mathrm{EW}}=\frac{\sqrt{2 \Delta \lambda \delta \lambda}}{\mathrm{S} / \mathrm{N}}$, where $\Delta \lambda$ is the integration range and $\delta \lambda$ is the spectral dispersion. The real errors might be larger due to unknown systematic uncertainties, particularly regarding the continuum normalisation. The $\mathrm{S} / \mathrm{N}$ was measured in the shaded region, and is shown in Appendix A. The FWHM $(=2 \sqrt{2 \ln 2} \sigma)$ of each DIB was measured by fitting a Gaussian function to the absorption lines.

\subsection{Correlation between EW(6196) and EW(6613)}

In order to check the consistency of the measurements we show our data, together with literature data, for the strongly correlated DIBs at 6614 and $6696 \AA$ in Fig. 4 (McCall et al. 2010; Krełowski et al. 2016). The dashed line shows a linear fit to the data by McCall et al. (2010) and the grey region shows the $1 \sigma$ error on the fit. The measured ratios are in agreement with the trend observed in other Galactic sightlines.

\section{3. $C_{60}^{+}$bands}

We measured the $\mathrm{C}_{60}^{+}$bands at 9577 and $9632 \AA$. The EW of the 9577 DIB increases roughly with $A_{V}$. B331 is an outlier with relatively weak 9577 and $9632 \AA$ DIBs. The average measured $9632 / 9577$ ratio is 1.1 , which is higher than the ratio 0.8 expected from laboratory measurements. The $9632 \AA$ DIB is known to suffer from contamination by a photospheric $\mathrm{Mg}$ II line. However, unless there are other factors impacting this ratio in M17, the stellar contamination has to be, on average, higher than $150 \mathrm{~m} \AA$ in order to reduce the observed ratio to 0.8 . This appears to require a rather large contribution from photospheric Mg II and we note that we cannot exclude systematic errors originating from telluric residuals. 
Table 3. DIB properties in the lines of sight to M17.

\begin{tabular}{|c|c|c|c|c|c|c|c|c|c|c|c|}
\hline$\lambda$ & B111 & B164 & B215 & B243 & B253 & B268 & B275 & B289 & B311 & B331 & B337 \\
\hline \multirow{2}{*}{4430} & 1.94 & 2.1 & 2.93 & 1.69 & 2.02 & 1.89 & 1.8 & 2.33 & 2.31 & - & - \\
\hline & $2027 \pm 32$ & $2539 \pm 145$ & $4794 \pm 218$ & $1779 \pm 240$ & $2020 \pm 158$ & $1742 \pm 130$ & $1919 \pm 36$ & $3304 \pm 311$ & $2528 \pm 53$ & - & - \\
\hline \multirow{2}{*}{5780} & 0.23 & 0.22 & 0.24 & 0.23 & 0.21 & 0.27 & 0.25 & 0.24 & 0.23 & - & - \\
\hline & $558 \pm 12$ & $521 \pm 38$ & $772 \pm 34$ & $540 \pm 45$ & $507 \pm 38$ & $663 \pm 49$ & $558 \pm 17$ & $711 \pm 49$ & $596 \pm 24$ & - & - \\
\hline \multirow{2}{*}{5797} & 0.11 & 0.14 & - & - & - & 0.26 & 0.17 & - & 0.15 & - & - \\
\hline & $89 \pm 8$ & $129 \pm 32$ & - & - & - & $185 \pm 30$ & $125 \pm 8$ & - & $168 \pm 8$ & - & - \\
\hline \multirow{2}{*}{6196} & 0.09 & 0.08 & 0.07 & 0.08 & 0.09 & 0.06 & 0.1 & - & 0.09 & - & - \\
\hline & $60 \pm 6$ & $62 \pm 12$ & $60 \pm 11$ & $58 \pm 13$ & $51 \pm 13$ & $55 \pm 13$ & $64 \pm 8$ & - & $63 \pm 6$ & - & - \\
\hline \multirow{2}{*}{6284} & 0.35 & 0.29 & 0.42 & 0.25 & 0.26 & 0.33 & 0.27 & 0.35 & 0.28 & - & - \\
\hline & $1691 \pm 11$ & $1790 \pm 24$ & $2206 \pm 28$ & $1720 \pm 34$ & $1708 \pm 38$ & $1580 \pm 29$ & $1661 \pm 36$ & $1920 \pm 57$ & $1770 \pm 25$ & - & - \\
\hline \multirow{2}{*}{6379} & 0.1 & 0.09 & 0.13 & 0.2 & 0.08 & 0.07 & 0.08 & 0.08 & 0.09 & - & - \\
\hline & $35 \pm 12$ & $51 \pm 10$ & $80 \pm 15$ & $54 \pm 24$ & $35 \pm 13$ & $32 \pm 8$ & $47 \pm 5$ & $65 \pm 10$ & $64 \pm 12$ & - & - \\
\hline \multirow{2}{*}{6614} & 0.14 & 0.14 & 0.13 & 0.13 & 0.13 & 0.14 & 0.14 & 0.14 & 0.13 & - & - \\
\hline & $188 \pm 6$ & $203 \pm 27$ & $251 \pm 12$ & $187 \pm 19$ & $168 \pm 15$ & $201 \pm 20$ & $203 \pm 27$ & $243 \pm 29$ & $227 \pm 11$ & - & - \\
\hline \multirow{2}{*}{7224} & 0.16 & 0.15 & 0.18 & 0.17 & 0.15 & 0.17 & 0.18 & 0.15 & 0.17 & 0.16 & 0.14 \\
\hline & $313 \pm 10$ & $287 \pm 12$ & $415 \pm 11$ & $386 \pm 9$ & $305 \pm 16$ & $339 \pm 12$ & $298 \pm 10$ & $337 \pm 15$ & $376 \pm 11$ & $387 \pm 28$ & $373 \pm 233$ \\
\hline \multirow{2}{*}{8620} & - & 0.53 & 0.48 & 0.47 & 0.42 & 0.49 & 0.44 & 0.43 & 0.55 & 0.48 & 0.54 \\
\hline & - & $562 \pm 17$ & $471 \pm 14$ & $485 \pm 14$ & $370 \pm 12$ & $493 \pm 12$ & $400 \pm 12$ & $457 \pm 19$ & $506 \pm 5$ & $494 \pm 16$ & $630 \pm 20$ \\
\hline \multirow{2}{*}{9577} & 0.44 & 0.45 & 0.48 & 0.47 & 0.55 & 0.45 & 0.42 & 0.45 & 0.45 & 0.4 & 0.47 \\
\hline & $371 \pm 14$ & $579 \pm 11$ & $546 \pm 22$ & $479 \pm 13$ & $512 \pm 20$ & $435 \pm 11$ & $439 \pm 10$ & $526 \pm 13$ & $632 \pm 17$ & $328 \pm 23$ & $489 \pm 16$ \\
\hline \multirow{2}{*}{9632} & 0.38 & 0.39 & 0.46 & 0.42 & 0.39 & 0.46 & 0.4 & 0.38 & 0.36 & 0.29 & 0.52 \\
\hline & $401 \pm 33$ & $586 \pm 26$ & $636 \pm 42$ & $499 \pm 34$ & $509 \pm 47$ & $531 \pm 25$ & $513 \pm 27$ & $550 \pm 24$ & $649 \pm 27$ & $436 \pm 31$ & $613 \pm 39$ \\
\hline \multirow{2}{*}{11797} & 0.32 & 0.27 & 0.32 & 0.39 & 0.31 & 0.36 & 0.21 & 0.27 & 0.26 & 0.64 & 0.99 \\
\hline & $278 \pm 13$ & $262 \pm 14$ & $205 \pm 15$ & $306 \pm 33$ & $269 \pm 29$ & $336 \pm 18$ & $173 \pm 44$ & $255 \pm 20$ & $261 \pm 14$ & $318 \pm 31$ & $457 \pm 49$ \\
\hline \multirow{2}{*}{13176} & 0.55 & 0.62 & 0.67 & 0.67 & 0.62 & 0.64 & 0.35 & 0.54 & 0.53 & 0.53 & 0.73 \\
\hline & $923 \pm 82$ & $1134 \pm 47$ & $1161 \pm 56$ & $1115 \pm 108$ & $1044 \pm 84$ & $1016 \pm 76$ & $463 \pm 70$ & $928 \pm 71$ & $859 \pm 53$ & $796 \pm 60$ & $1635 \pm 88$ \\
\hline \multirow{2}{*}{15268} & 0.46 & 0.71 & 0.58 & - & 0.74 & 0.57 & - & 0.52 & 0.51 & - & - \\
\hline & $395 \pm 34$ & $875 \pm 25$ & $521 \pm 42$ & - & $615 \pm 47$ & $820 \pm 42$ & - & $438 \pm 18$ & $401 \pm 17$ & - & - \\
\hline
\end{tabular}

Notes. Column 1 shows the rest wavelength of the DIBs. Columns 2-12 show the FWHM (nm; first row) and EW (mÅ; second row) measured for the sightlines towards the objects in M17.

In the optically thin limit the EW of the $9577 \AA$ DIB can be converted into a column density. We do not use the $9632 \AA$ DIB as it is known to suffer from photospheric line contamination which is difficult to correct for. For the observed $9577 \AA$ DIB strengths this gives $\mathrm{N}\left(\mathrm{C}_{60}^{+}\right)=2.5-4.3 \times 10^{13} \mathrm{~cm}^{-2}$. These are somewhat lower abundances than the neutral $\mathrm{C}_{60}$ abundance of $2.4 \times 10^{14} \mathrm{~cm}^{-2} / G_{0}$, where $G_{0}$ is the radiation field, measured with Spitzer in the diffuse ISM (Berné et al. 2017). However, the higher sensitivity of JWST opens the possibility to detect fullerene infrared emission (potentially both $\mathrm{C}_{60}$ and $\mathrm{C}_{60}^{+}$features) towards M17 that would allow a comparison between emission and absorption measurements in the same environment.

\subsection{DIB profiles}

Dahlstrom et al. (2013) reported on anomalous DIB profiles in the sightline towards Herschel 36. According to the authors, the profiles of the 5780, 5797, 6196, and 6614 DIBs show an extended red wing in comparison to nominal sightlines. Oka et al. (2013) confirmed this finding for three of the DIBs, but did not detect a pronounced red wing in the DIB at $6196 \AA$ (we also do not detect a red wing in the profile of the 6196 DIB with the lower-resolution $\mathrm{X}$-shooter data). These authors performed a study that allowed them to make a distinction between the carriers of the above DIBs at 5780, 5797, and 6614 DIBs and others (e.g. 5850, 6196, and 6379) which do not present such a pronounced red wing.

In addition, the sightline towards Herschel 36 shows absorption lines from rotationally excited $\mathrm{CH}^{+}$and $\mathrm{CH}$ and from vibrationally excited $\mathrm{H}_{2}$. Furthermore, an atypical extinction curve is observed with $R_{V} \sim 6$ and a weak $2175 \AA$ bump, i.e. a flat far-UV extinction curve (Dahlstrom et al. 2013). M17 also shows anomalous extinction $\left(R_{V}>3.1\right.$; Paper I); therefore, it is a good candidate to search for anomalous behaviour in the DIBs.

We compare the profiles of the DIBs towards M17 with those observed in the direction of Herschel 36 and HD 161061 (a nominal sightline) in Fig. 5. The X-shooter spectra of Herschel 36 (unpublished) and the nominal sightline (HD 161061) were obtained previously by us with ESO programs 091.C0934(B) and 385.C-0720(A). The spectra for these two sources were reduced using the same procedure described in Sect. 2.

To facilitate the comparison, we scaled the flux of the DIBs towards Herschel 36 and HD 161061 to match the depths of the cores of the DIBs towards M17. The profiles of the DIBs towards M17 are similar to the "nominal" profile, and they do not show a red wing like that in the case of Herschel 36. In the case of the $6196 \AA$ DIB we do not detect a red wing in the spectrum of Herschel 36. 


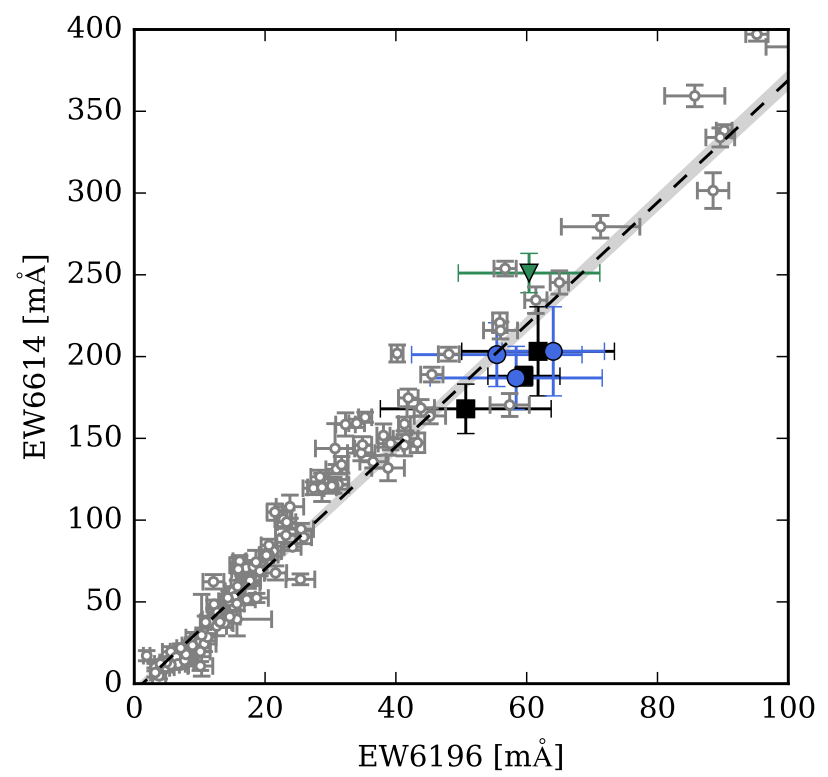

Fig. 4. Correlation between the DIBs at 6614 and $6196 \AA$. The dashed line represents the relation found by McCall et al. (2010; grey dots) and the grey area shows the $1 \sigma$ errors to the fit. The blue circles represent the sightlines towards objects with circumstellar disks, the green triangles towards stars with IR excess longwards of $2.5 \mu \mathrm{m}$, and the black squares towards OB stars.

\section{Comparison with other sightlines}

For reddened lines of sight the strength of many DIBs increases in a roughly linear way with $E(B-V)$. The relation between the DIB strength and the reddening is not very well constrained for high extinction values because of the lack of data covering those high values of $E(B-V)$. Also, these studies have been applied to only a few prominent DIBs, mostly the 5797, 5780, and $6614 \AA$ DIBs.

In Fig. 6, we compare the strength of some prominent DIBs in M17 as a function of the reddening $E(B-V)$ with that in other published studies. We plot a linear fit to a large variety of Galactic sightlines (Chlewicki et al. 1986; Benvenuti \& Porceddu 1989; Herbig 1993; Sonnentrucker et al. 1997; Krełowski et al. 1999; Tuairisg et al. 2000; Rawlings et al. 2003; Galazutdinov et al. 2004; Cox et al. 2005; Friedman et al. 2011, for details see figure captions) excluding the starforming regions (M17, Herschel 36, and RCW 36) for the three DIBs mentioned above with dashed black lines. With the grey area we show the linear fit and the $1 \sigma$ error bars to the fit. For reference, we also plot DIB equivalent width measurements for Herschel 36 and for the massive star-forming region RCW 36 (Ellerbroek et al. 2013). The large scatter on these relations is intrinsic due to physical variations between lines of sight, and not due to measurement uncertainties. For example, Vos et al. (2011) show that there is a dichotomy in the DIB strength versus extinction relation for UV exposed and UV shielded environments.

The M17 points are located below the average Galactic trend, like the sightlines probing the dense Cyg OB2 region. On the other hand, when including the DIBs towards M17 in the fit, the slope of the relation does not change significantly for each of the three DIBs.

We also compared the strength of the NIR DIB at $15268 \AA$ as a function of $\mathrm{E}(B-V)$ with several Galactic sightlines taken
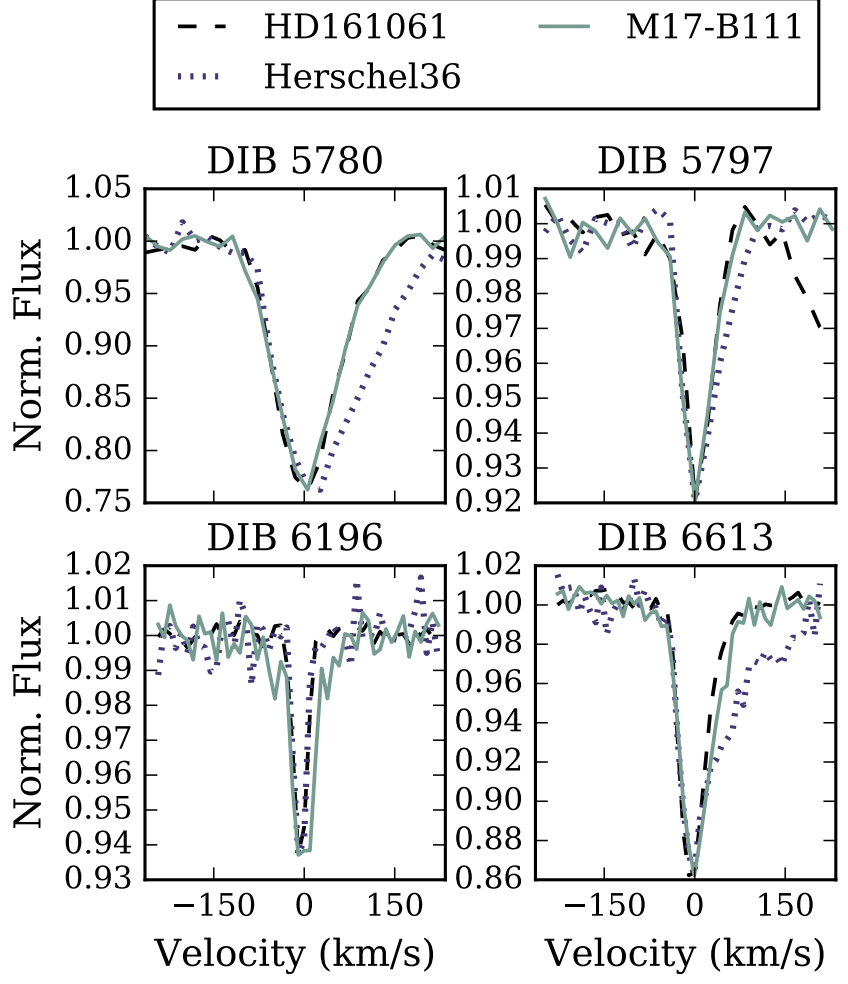

Fig. 5. Comparison of the shape of some DIBs towards M17 (B111, green solid line) with the sightline towards Herschel 36 (purple dotted line) and a "nominal" sightline (black dashed line). All the spectra have been convolved to the same resolution and scaled to have the same line central depth. The M17 DIB profiles do not show an extended red wing or any other anomaly with respect to the DIB in HD 161061.

from the SDSS-III APOGEE survey by Zasowski \& Ménard (2014). We do not show this comparison, but we observe that the sightlines towards M17 follow the same trend as those observed in different Galactic environments. We conclude that the DIB behaviour with respect to reddening $E(B-V)$, as observed in the direction of M17, indicates that these sightlines probe a denser ISM, but are not peculiar.

\subsection{Ratio of $E W(5797)$ to $E W(5780)$}

Vos et al. (2011) demonstrated that the ratio 5797/5780 might be useful to distinguish between sightlines probing diffuse cloud edges and denser cloud cores. The $5797 \AA$ DIB is less sensitive to UV radiation than the $5780 \AA \mathrm{DIB}$; therefore, we expect that the $5797 / 5780$ ratio would be lower in higher extinction environments. This effect is strongest in sightlines dominated by one velocity component in the interstellar gas absorption profile (i.e. single-cloud sightlines) and tends to get "washed-out" in multicloud sightlines. The EW ratio of the $5797 / 5780$ DIBs is plotted in Fig. 7. Compared to the lower extinction data $(E(B-V)<$ 1 mag; Friedman et al. 2011; Vos et al. 2011) the DIBs towards M17 cover a smaller range (0.15-0.45) in the 5797/5780 DIB ratio (though at higher extinction values). We do not find a clear correlation with spatial location in the cloud. We note that the interstellar gas absorption features of $\mathrm{Ca}$ II and $\mathrm{Na}$ I are largely unresolved at the spectral resolution of X-shooter. It is conceivable that at higher spectral resolution the interstellar gas component associated with M17 will be resolved in multiple velocity components. This could imply that due to averaging along the 

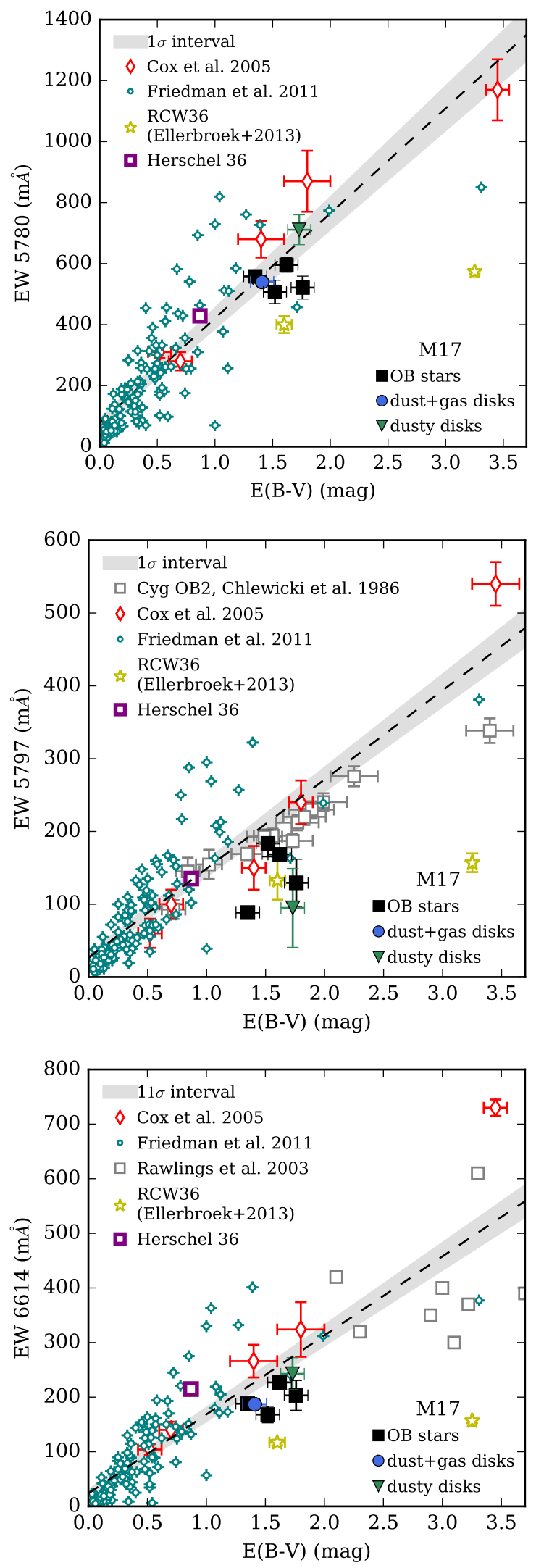

Fig. 6. Equivalent width of the 5780 (top panel), 5797 (middle panel), and $6614 \AA$ (bottom panel) DIBs as a function of reddening $E(B-V)$ for Galactic sightlines. The DIB strengths are taken from the literature (open symbols) and this work (filled symbols). The dashed line indicates the linear fit for the Galactic sightlines excluding the star-forming regions (RCW 36, Herschel 36, and M17), and the grey area represents the $1 \sigma$ error on the fit.

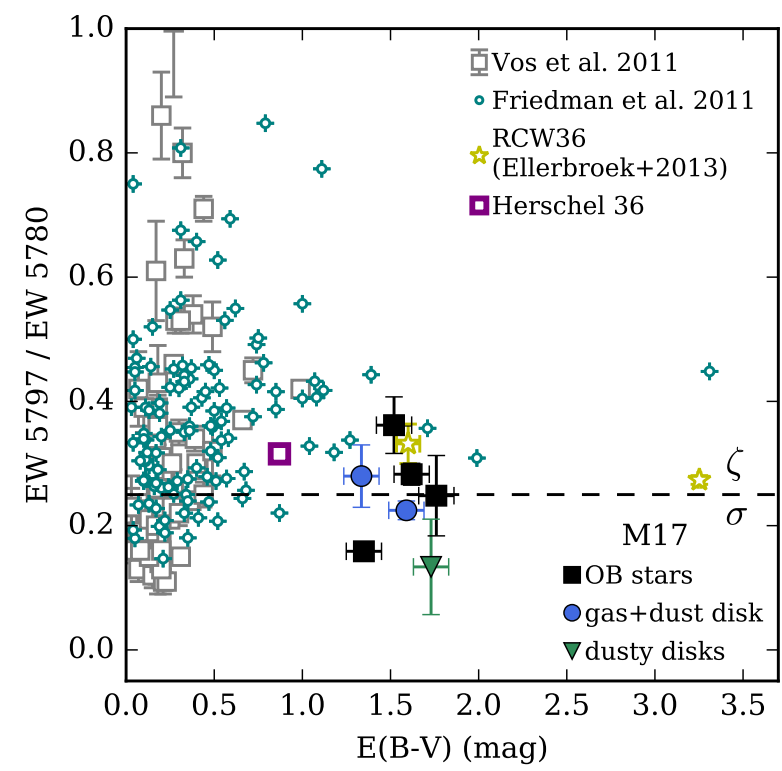

Fig. 7. Ratio of the 5797 to 5780 DIBs. The open symbols show data from the literature and the filled symbols data from the sightlines towards M17. The dashed line represents the division between $\zeta$ - and $\sigma$-type clouds.

line of sight the observed 5797/5780 ratio constitutes an average of this ratio in individual clouds. Variations of 5797/5780 (in diffuse ISM) are thought to depend strongly on the local geometry and the effective UV radiation field (see above), both of which are complex within the large H II region. Additionally, variations in the $5797 / 5780$ ratio in the densest regions (high $R_{V}$ ) can potentially be driven by different mechanisms (e.g. depletion) compared to changes in this ratio in the more diffuse medium (e.g. effective UV radiation field); this could be the case for M17 as the change on strength (per $A_{V}$ ) of these two DIBs with $R_{V}$ is different from each other as shown in Fig. 10 and discussed in Sect. 6.

\section{2. $8620 D I B$}

The DIB at $8620 \AA$ A has recently gained more attention because it has been included in the large-scale surveys of RAVE and Gaia. It has been shown that the EW of this DIB is well correlated with the colour excess $E(B-V)$; Munari et al. (2008) reported a relation between the DIB strength and $E(B-V)$ given by $E(B-V)=(2.72 \pm 0.03) \times \mathrm{EW} 8620 \AA$ for $E(B-V)<1.2$.

Maíz Apellániz et al. (2015) measured this DIB in two sightlines with high extinction towards $\mathrm{O}$ stars in the cluster Berkeley 90. The authors detect at least two clouds moving at different velocities towards these objects; one thinner cloud associated with the material far away from the stellar cluster, and a thicker one associated with the material local to the cluster. The latter cloud is thicker for one sightline, which allowed the authors to measure the properties of DIBs in $\sigma\left(R_{V} \sim 4.5\right)$ and in $\zeta\left(R_{V} \sim 3.1\right)$ clouds (see Sect. 5.1 for details about $\sigma$ and $\zeta$ clouds). They observed that the DIB at $8620 \AA$ is highly depleted in dense clouds and, therefore, correlates poorly with extinction higher than $A_{V} \sim 6$.

Damineli et al. (2016) extended the correlation of the 8620 DIB with extinction by adding sightlines with higher values of $A_{K}$. They used the extinction in the $K$ band because it is less sensitive to the grain size than $A_{V}$. They adopted 


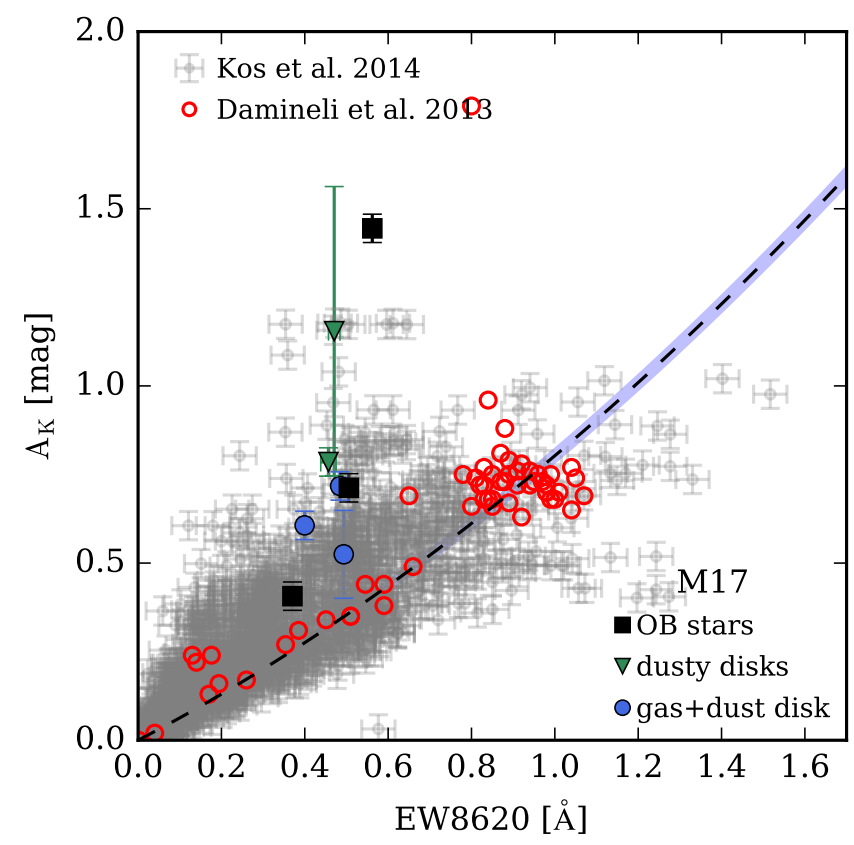

Fig. 8. $K$-band extinction $A_{K}$ vs. strength of the DIB at $8620 \AA$. The open symbols show data from the literature, and the filled symbols sightlines towards M17. The dotted line and the blue area show the relation in Eq. (2).

$A_{K s}=0.29 E(B-V)$, which allowed the Munari et al. (2008) relation to be expressed as $A_{K s}=(0.691) \times E W 8620 \AA$. They find the following correlation:

$A_{K s}=(0.612 \pm 0.013) E W+(0.191 \pm 0.025) E W^{2}$.

This is in good agreement with Munari et al. (2008) for $E W<0.6 \AA$, which translates to $A_{V}<9$ mag for the extinction properties of their sample.

Using data from the RAVE survey, Kos et al. (2014) measured the strength of the $8620 \AA$ DIB for $\sim 500000$ spectra at distances $<3 \mathrm{kpc}$. We plot the EW of the $8620 \AA$ DIB in Fig. 8 . We include the data by Damineli et al. (2016, red dots) and Kos et al. (2014, grey dots), the black dashed line and the blue shadow represent the relation in Eq. (2). In order to derive $A_{K}$ from the Kos et al. (2014) data, we adopted $A_{K} / A_{V}=0.11$.

The DIB strength towards M17 shows a very large spread in comparison with the relation by Damineli et al. (2016). This spread is similar to that observed in the sightlines from the RAVE survey. The large scatter in the M17 data could be an indication that the DIB carrier becomes depleted at extinctions $A_{K}>1.0$. We detect a general positive correlation between the strength of the $8620 \mathrm{DIB}$ and the $K$-band extinction for $A_{K} \lesssim 1.0$.

\section{DIB strengths versus $\boldsymbol{R}_{V}$}

The value of $R_{V}$ is related to the size distribution of the dust grains. High values of $R_{V}$ imply a relative over-abundance of large grains, whereas low $R_{V}$ values indicate that the small dust grains are more dominant in the size distribution. Typically higher values of $R_{V}$ are associated with denser clouds where grain growth is or has been more significant than in diffuse regions. This is reflected in the relative slope of the extinction curve. Higher $R_{V}$ values correspond to a flatter extinction curve,

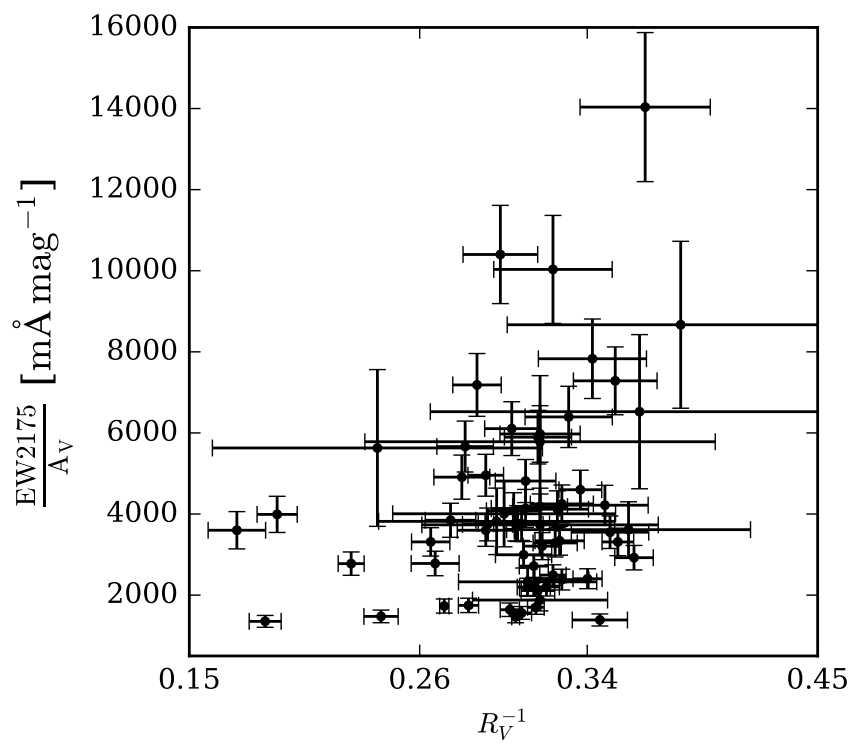

Fig. 9. Normalised EW of the $2175 \AA$ feature measured in Galactic sightlines as a function of $R_{V}$. The measurements are obtained from Xiang et al. (2017). The $2175 \AA$ feature is stronger in sightlines characterised by a lower value of $R_{V}$.

meaning that photons at all wavelengths are more equally absorbed.

Cardelli et al. (1989) presented observational evidence that the $2175 \AA$ feature, normalised by the amount of extinction, shows a positive trend with $R_{V}^{-1}$. In Fig. 9 we present a similar plot based on measurements recently collected by Xiang et al. (2017). Although the plot reveals significant scatter, there appears to be a general trend that sightlines with a lower value of $R_{V}$ show a stronger $2175 \AA$ feature per magnitude visual extinction. The Pearson correlation coefficient between $\operatorname{EW}(2175 \AA)$ and $R_{V}^{-1}$ has a value of $r=0.3$, which indicates a rather weak formal correlation.

In Fig. 10 we plot the strength of the DIBs normalised by the visual extinction $A_{V}$ with $R_{V}^{-1}$. We include the sightlines towards M17, Herschel 36, and the object 408 in RCW 36. Where available, we add the data from Xiang et al. (2017) who compiled the EW of several DIBs in 97 sightlines. For most DIBs we discern a positive trend of the normalised DIB strength $\left(\mathrm{EW} / A_{V}\right)$ with $R_{V}^{-1}$, similar to the trend seen for the $2175 \AA$ feature (Fig. 9). Given that $A_{V}$ is in the denominator of both axes, if the uncertainties in $A_{V}$ are large, a spurious relation between $\mathrm{EW} / A_{V}$ and $R_{V}^{-1}$ might result. This is because when dividing by a very uncertain $A_{V}$, we expect some values of both EW/A $A_{V}$ and $R_{V}^{-1}$ to be very low and some to be very large causing a spurious linear relation intercepting at zero. However, we consider the errors in $A_{V}$ to be small enough - primarily due to the accurate determination of the spectral type - that we do not expect such an effect here.

We note that there is a cloud of data points centred at the Galactic average value of $R_{V}=3.1$. The observed scatter could represent sampling of non-heterogeneous (multiple) interstellar cloud conditions in these sightlines. In other words, variations in DIB band strength (per unit reddening) are more pronounced than variations in average dust grain size. The presence of this cloud of data points shows that normalising by $A_{V}$ does not introduce a spurious relation. If that were the case, we would expect the points at all $R_{V}$ values to follow a linear relation. On the other hand, for sightlines with $R_{V} \gtrsim 3.8\left(R_{V}^{-1} \lesssim 0.26\right)$ there appears to be a much more pronounced (linear) relation between $\mathrm{EW} / A_{V}$ 
Table 4. Results of the linear fit of EW/A $A_{V}$ vs. $R_{V}^{-1}$ towards M17 sightlines.

\begin{tabular}{cccc}
\hline \hline DIB & $\begin{array}{c}\text { Slope } \\
\left(\mathrm{m \AA} \mathrm{mag}^{-1}\right)\end{array}$ & $\begin{array}{c}\text { Interception } \\
\left(\mathrm{m \AA} \mathrm{mag}^{-1}\right)\end{array}$ & Pearson $r$ \\
\hline 4430 & $1604 \pm 403$ & $-38 \pm 97$ & 0.39 \\
5780 & $868 \pm 195$ & $-115 \pm 45$ & 0.80 \\
5797 & $375 \pm 110$ & $-67 \pm 26$ & 0.63 \\
6196 & $62 \pm 24$ & $-5 \pm 5$ & 0.91 \\
6284 & $1705 \pm 324$ & $-137 \pm 76$ & 0.85 \\
6379 & $17 \pm 13$ & $3 \pm 3$ & 0.21 \\
6614 & $211 \pm 56$ & $-18 \pm 13$ & 0.88 \\
7224 & $499 \pm 99$ & $-67 \pm 23$ & 0.70 \\
8620 & $594 \pm 130$ & $-68 \pm 30$ & 0.69 \\
11797 & $500 \pm 106$ & $-75 \pm 24$ & 0.46 \\
13176 & $1714 \pm 462$ & $-254 \pm 107$ & 0.11 \\
15268 & $1992 \pm 551$ & $-372 \pm 128$ & 0.63 \\
9577 & $501 \pm 107$ & $-41 \pm 25$ & 0.62 \\
9632 & $573 \pm 121$ & $-53 \pm 28$ & 0.82 \\
\hline
\end{tabular}

and $R_{V}^{-1}$. The M17 data presented here strengthen the presence of this linear relation that can already be gleaned from the data compiled by Xiang et al. (2017) where most of the sightlines with $R_{V} \gtrsim 3.8$ correspond to stars in star-forming regions (Orion giant molecular cloud, Upper Scorpius, Triffid nebula, and Rho Ophiuchi).

We performed an orthogonal distance regression (ODR; Churchwell 1990) $)^{5}$ to fit a linear function to the DIB strength normalised by the visual extinction $A_{V}$ versus $R_{V}^{-1}$ for sightlines towards M17. The fit results, together with the Pearson correlation coefficients are listed in Table 4 and shown in Fig. 10, where the black line corresponds to the best fit to the M17 data alone and the shaded region to the $1 \sigma$ error on the fit.

Based on the results from the fit, we confirm that for M17 there is a linear relation between the normalised DIB strength and $R_{V}^{-1}$. This correlation is strongest for the DIBs at 5780, 6196, 6284, 6614, and $9632 \AA$. From the Pearson correlation coefficient, $r$, we find that the correlation is moderate to strong $(r>0.6)$ for all DIBs except for those at 4430, 6379, 11797, and $13176 \AA$ where the correlation is weak $(r<0.5)$. We note that for the 11797 and $13176 \AA$ DIBs there is a clear outlier data point which strongly affects the Pearson correlation coefficient $r$ (Fig. 10). This point corresponds to the B275 sightline where the spectral quality in the ranges of these two DIBs is poor, which could introduce systematic errors in the DIB measurements (see Figs. A.12 and A.13). Excluding this point results in $r>0.7$ for both DIBs.

\section{Discussion and conclusion}

\subsection{Extinction in M17}

We calculated the extinction properties of the sightlines towards M17, with overall relatively high $R_{V}$ values, using different methods: $i$ ) by dereddening the SED of the stars using Cardelli's extinction law and then fitting it to Kurucz models to find $A_{V}$ and $R_{V}$ (from that $\left.E(B-V)=A_{V} / R_{V}\right) ; i i$ ) by directly comparing the intrinsic magnitudes to the observed values to find $A_{V}$ and

\footnotetext{
5 Information about the python package is available at https:// docs.scipy.org/doc/scipy/reference/odr.html
}

$E(B-V)\left(R_{V}=A_{V} / E(B-V)\right)$; and iii) by finding $A_{V}$ and $E(B-V)$ from the intrinsic and observed magnitudes and calculating $R_{V}$, as defined by Fitzpatrick \& Massa (2007; Eq. (1)). We observe that the colour excess $E(B-V)$ calculated with Cardelli's extinction law and the SED fitting is systematically higher than when using the other two methods. This confirms that this extinction law is not well suited for high values of $R_{V}$, as already stated in Cardelli et al. (1989). We conclude that the best way of obtaining the extinction properties is to use the intrinsic and observed magnitudes and then calculate $R_{V}$ as shown in Eq. (1), because with this equation it is also possible to take into account the NIR part of the SED.

\subsection{Relation of DIBs with $E(B-V)$}

In Fig. 6, we show the relation of the DIBs at 5780 and $5797 \AA$ with the colour excess $E(B-V)$ in M17 and compare it with other sightlines published in the literature. The value of $E(B-V)$ for the sightlines in M17 is relatively high compared to other Galactic sightlines; nevertheless, the DIB strength seems to follow the same trend as observed in other reddened sightlines.

Ellerbroek et al. (2013) reported an anomalous behaviour of the DIB strength in sightlines towards the star-forming region RCW 36. We do not find a similar deviation in the sightlines towards M17. The behaviour reported in RCW 36 could be due to the way in which the authors calculated $A_{V}$. They assumed a value of $R_{V}=3.1$, dereddened the spectrum using Cardelli's extinction law, and then fitted it to Kurucz models. In this paper we show that the value of $E(B-V)$ can be overestimated by as much as 0.5 mag (Fig. 2) when using Cardelli's extinction law. Previous studies have shown that star-forming regions such as M17 and M8 (where Herschel 36 resides) often show high values of $R_{V}$ (e.g. Cardelli et al. 1989; Hanson et al. 1997). Therefore, the assumption that $R_{V}=3.1$ for RCW 36 probably underestimates its true $R_{V}$.

\subsection{Relation of DIBs with $R_{V}$}

The observed trend of EW(DIB)/ $/ A_{V}$ with $R_{V}^{-1}$ for M17 and other sightlines with $R_{V} \gtrsim 3.8$ ( $R_{V}^{-1} \lesssim 0.26$; cf. Fig. 10$)$ is reminiscent of what Cardelli et al. (1989) and Xiang et al. (2017) find for the $2175 \AA$ bump, the carrier of which is thought to consist of some (unidentified) carbonaceous material; however, the trend of the bump is weaker than that observed for the DIBs.

The slope of the linear relation between EW/A $A_{V}$ and $R_{V}^{-1}$ (valid for $R_{V} \gtrsim 3.8$ ) can be interpreted as a measure of the sensitivity to which variations in the abundance of DIB carriers (per unit visual extinction) are related to variations in the average grain size of interstellar dust. This slope can then be interpreted in the context of either the depletion of the DIB carrier as the dust grains coagulate and grow (see e.g. Jura 1980; Ormel et al. 2009, EW/ $/ A_{V}$ decreases while $R_{V}$ increases) or the production of DIB carriers as the larger grains get destroyed by the increasingly effective UV radiation field (see e.g. Cecchi-Pestellini et al. 1995; Jones 2004, EW/ $A_{V}$ increases and $R_{V}$ decreases).

Alternatively, in the hypothesis that some DIB carriers are ionised species, the strengths of DIBs are expected to increase with a stronger effective UV radiation field. If $R_{V}$ could be regarded as a tracer of the effective UV radiation field, higher values correspond to a lower ionisation fraction, hence lower $\mathrm{EW} / A_{V}$. In this context the slope of $\mathrm{EW} / A_{V}$ versus $R_{V}^{-1}$ would be directly related to the ionisation potential of the carrier species.

With the current data we cannot distinguish between these different scenarios. However, it is possible to identify groups or 

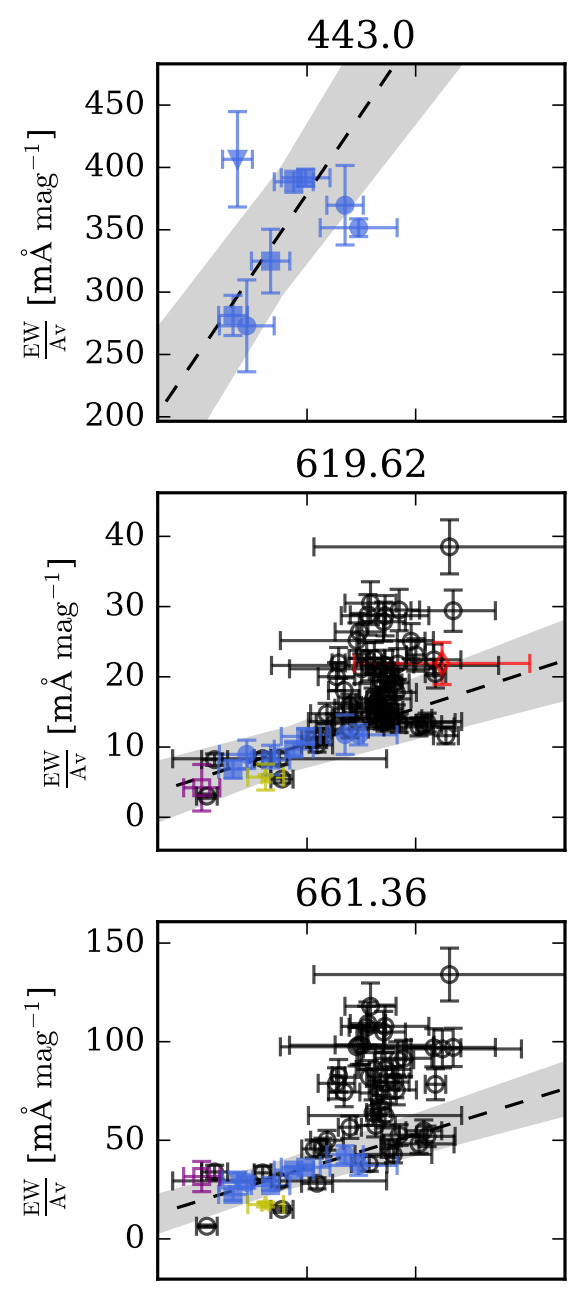

1179.7
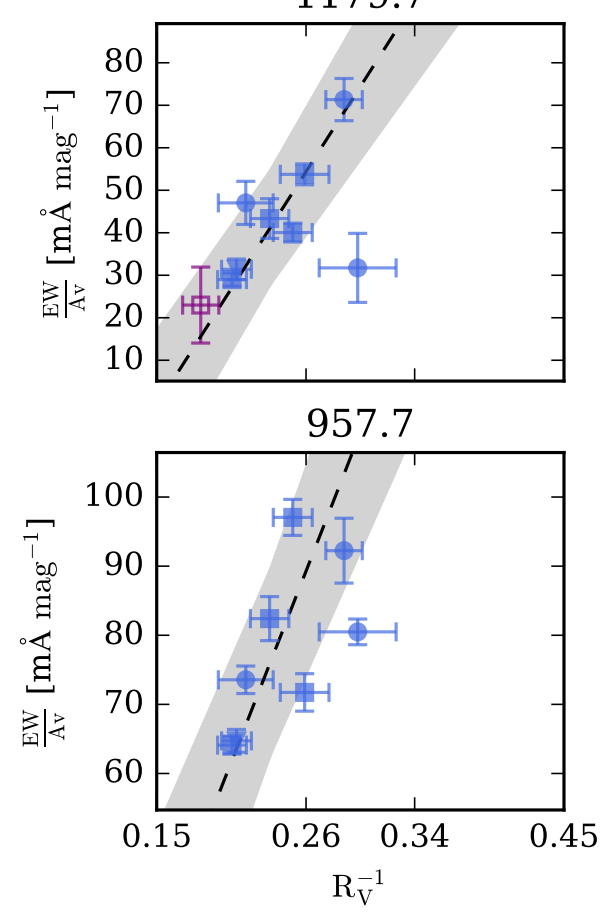

578.05

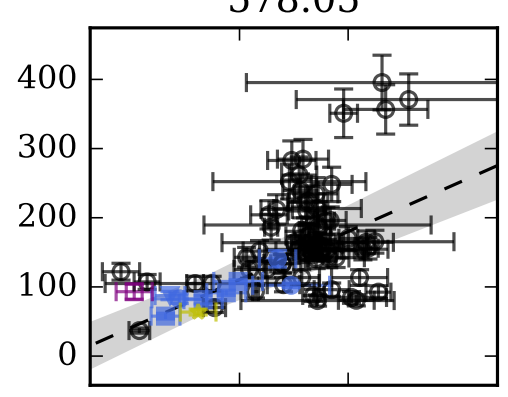

628.4

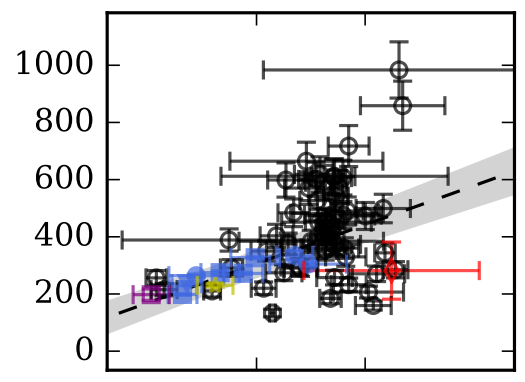

722.4

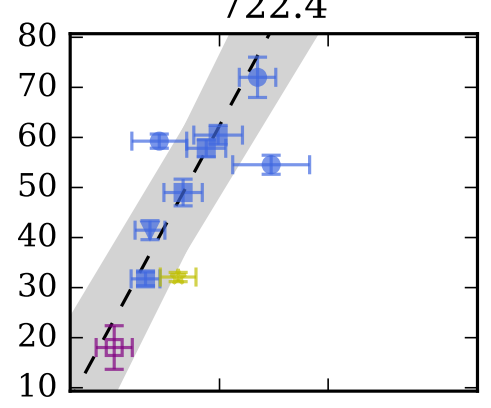

1317.6

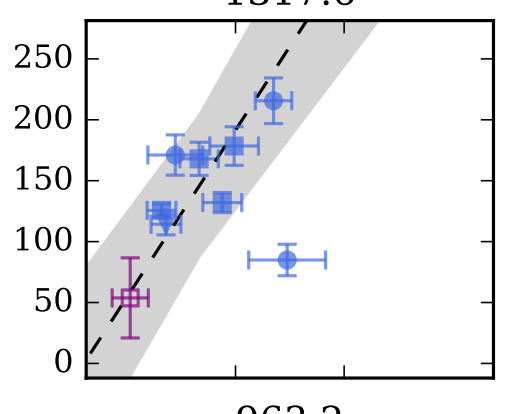

963.2

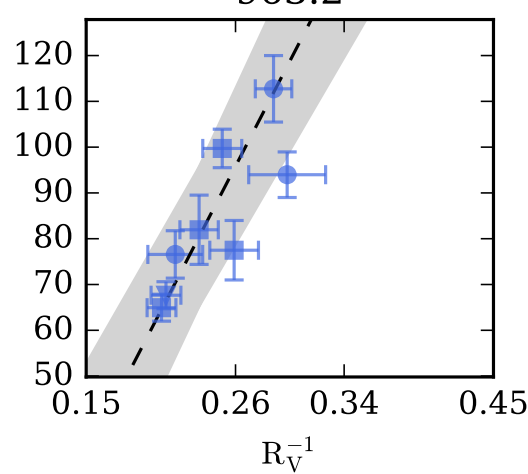

579.7

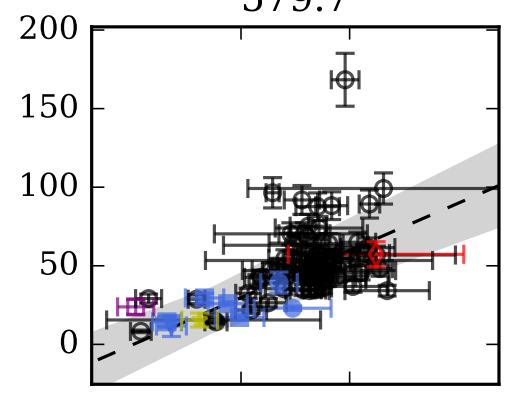

637.9

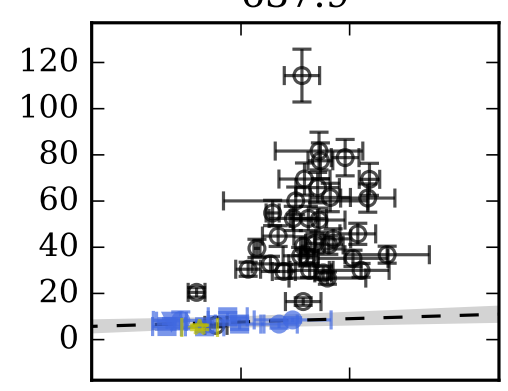

862.0

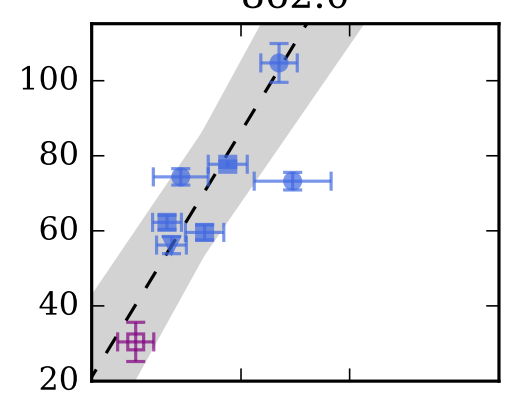

1526.8

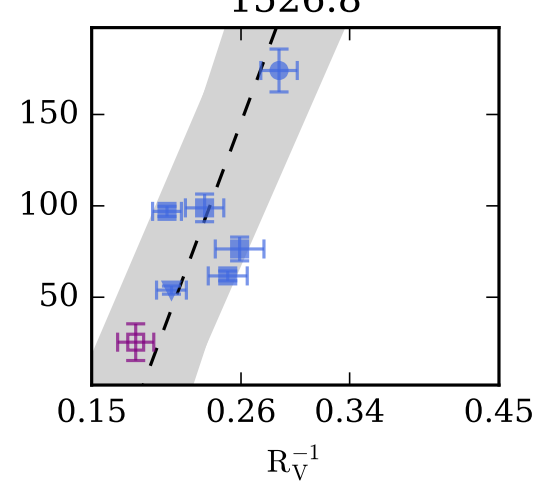

Fig. 10. Normalised DIB EW vs. $R_{V}^{-1}$. The blue symbols show the M17 data; the dots show the stars in M17 with circumstellar disks, the triangles the stars with an IR excess longwards of $3 \mu \mathrm{m}$, and the squares the OB stars in M17. The open symbols show data from the literature. The purple square shows the position of Herschel 36, the yellow star shows the source 408 in RCW 36 , and the red diamond shows the high mass X-ray binary 4U1907+09. The grey dots show the data by Xiang et al. (2017) when available. The black line shows a linear fit with $R_{V}^{-1}$ and the shaded region the $1 \sigma$ error box of the fit to the M17 measurements only. 

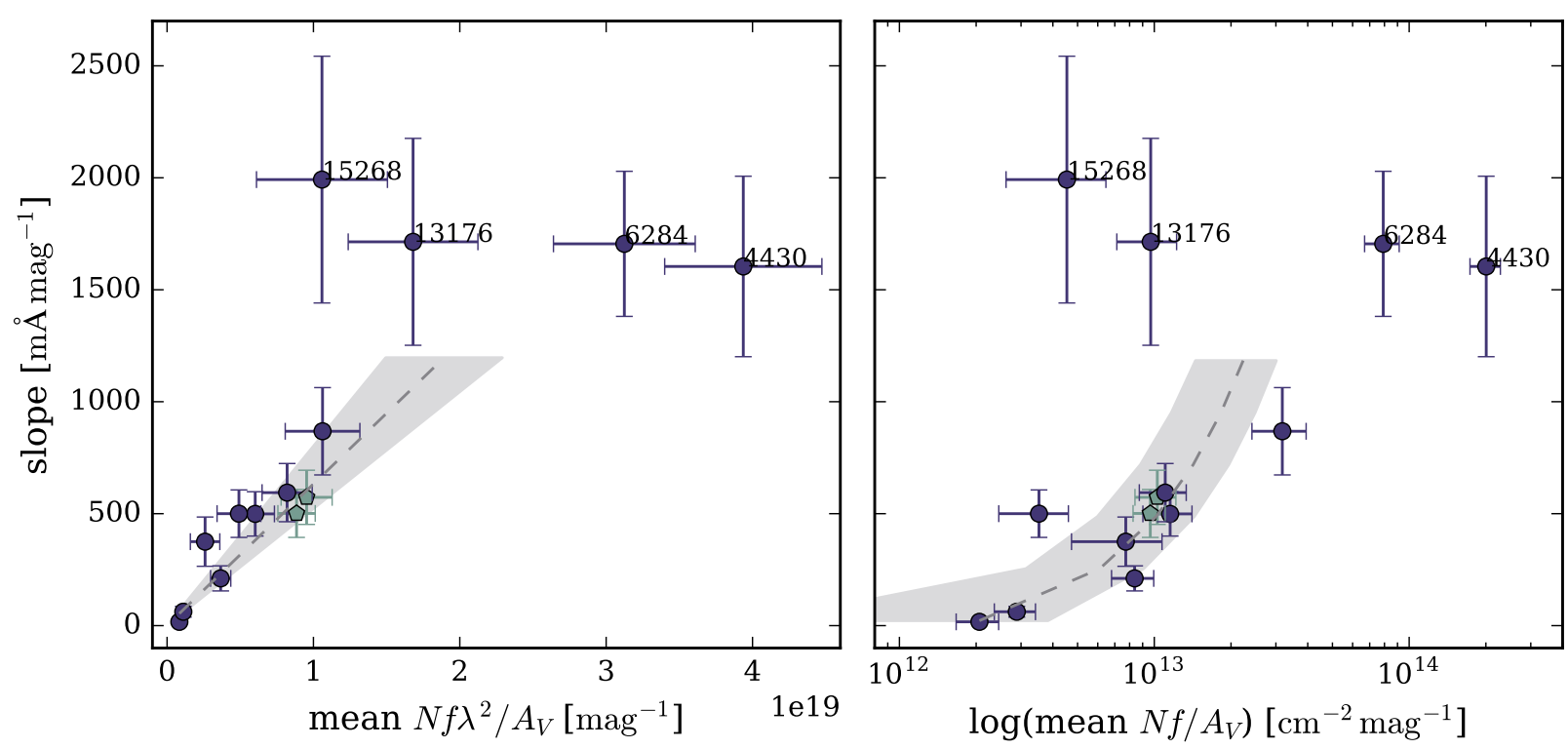

Fig. 11. Slope representing the sensitivity of EW/A $A_{V}$ with changes in $R_{V}^{-1}$ vs. mean $A_{V}$ normalised line strength (left panel, expressed as $\left.N f / A_{V}\right)$ and vs. the log of this quantity (right panel) for all sightlines. The dashed line and the shaded regions represent the linear fit to the group of DIBs with slopes $\lesssim 1000$ and the $2 \sigma$ error bars to the fit. The two $C_{60}^{+}$DIBs are represented with light green pentagons.

families of DIB carriers which behave similarly in the transition from the more dense to diffuse ISM (high to low $R_{V}$ ). To investigate this we compared the column density of the DIB carriers multiplied by the (unknown) oscillator strength, $N f$, with the slope $\mathrm{d}\left(\mathrm{EW} / A_{V}\right) / \mathrm{d} R_{V}^{-1}$ (cf. Fig. 10; Table 4). This slope can be viewed as a measure of the sensitivity of the DIB carriers to changes in $R_{V}^{-1}$, hence in grain size distribution or as a measure of the carrier's ionisation potential. We refer to this slope as the DIB $-R_{V}$ sensitivity. Assuming that the optically thin approximation holds for the DIBs (no saturation of absorption) we have

$N f=1.13 \times 10^{20} \frac{E W}{\lambda^{2}}$,

where $N$ is the column density in $\mathrm{cm}^{-2}, f$ the oscillator strength, EW is the equivalent width (in $\AA$ ) and $\lambda$ is the central wavelength of each DIB (in $\AA$ ). We show the dependence of the DIB- $R_{V}$ sensitivity on $N f \lambda^{2}$, and $N f$ in Fig. 11; In both cases a linear fit is shown with a dashed line and the shaded region shows the $2 \sigma$ error to the fit. Additionally, we marked the $C_{60}^{+}$DIBs with green pentagons.

First we discuss the results in the context of $R_{V}$ as a proxy for changes in the grain size distribution. We are able to identify two groups; the first group contains ten moderately strong DIBs: the two $C_{60}^{+}$DIBs, the DIBs presenting a red wing in Herschel 36 (5780, 5797, and 6614), and those at 6196, 6379, 7224, 8620, and $11797 \AA$. These DIBs show a modest sensitivity to the change in grain size, and their sensitivity increases almost linearly as a function of DIB strength. The second group consists of four strong DIBs (at 4430, 6284, 13176, and $15268 \AA$ ) that are very sensitive to the change in grain size distribution (large $\mathrm{d}\left(\mathrm{EW} / A_{V}\right) / \mathrm{d} R_{V}^{-1}$ slope values), but their DIB- $R_{V}$ sensitivity does not seem to depend on the DIB strength.

In the case of the first group, we postulate that the observed linear increase in the DIB- $R_{V}$ sensitivity with the amount of DIB carriers, $N f$, suggests that the increase in the latter is primarily due to an increase in column density and that $f$ is quite similar for this group of DIB carriers. This is because the reverse situation, i.e. that a systematic increase in the sensitivity of DIB carriers to changes in $R_{V}$ is related to a systematic increase in the oscillator strength of the DIB carriers appears much more unlikely. If this is the case, the oscillator strengths of these DIBs could be extracted with reference to the two DIBs at 9577 and $9632 \AA$ assigned to $\mathrm{C}_{60}^{+}\left(f_{9577}=0.018\right.$ and $\left.f_{9632}=0.015\right)$.

The linear dependency of the DIB- $R_{V}$ sensitivity on the DIB strength implies that the relative production/destruction rate of these DIB carriers is the same for the same change in typical grain size $\left(R_{V}\right)$. In other words, for a doubling of the abundance of DIB carriers in the first group, the same change in grain size distribution is required. This may imply a common production/destruction mechanism. The fact that the DIB- $R_{V}$ sensitivity of the stronger DIBs is independent of their strength suggests the existence of an additional reservoir of DIB carriers unrelated to the dust grains. These carriers would be produced or destroyed as the grain size distribution changes and via other mechanisms.

Alternatively, we consider the scenario where $R_{V}$ is a proxy of the effective UV radiation field strength and the DIB carriers are large ionised molecules. In this case Fig. 11 has to be interpreted differently. Here the slope $\mathrm{d}\left(\mathrm{EW} / A_{V}\right) / \mathrm{d} R_{V}^{-1}$ is an indirect measure of the carrier's ionisation potential. The broad DIBs at $15268,13176,6284$, and $4430 \AA$ have a (similar) strong slope which implies a (similar) low ionisation potential. The ionisation potential of the carriers of these broad DIBs is then expected to be significantly lower than the ionisation potential of $7.58 \mathrm{eV}$ measured for $C_{60}$ (de Vries et al. 1992). In the opposite direction, the narrow DIBs at 7224, 5797, 6614, 6196, and $6379 \AA$ which have increasingly shallower slopes should then have increasingly higher ionisation potentials. For PAHs the ionisation potential is strongly related to their number of $\pi$-electrons (see Fig. B.1. in Ruiterkamp et al. 2005).

We note that in the case that there are several foreground clouds contributing to the extinction in the sightline towards M17, the interpretation of the observed relations is complicated. The hypotheses presented above assume that most of the variation in $A_{V}$ and $R_{V}$ arises from the dust and gas in the M17 region. 
Correcting for a foreground sheet of dust, with $A_{V}=2$ and a constant foreground $\mathrm{EW}(\mathrm{DIB}) / A_{V}$ contribution projected across the M17 region, would shift all the $\mathrm{EW} / A_{V}$ values vertically. Also, correcting for foreground dust with a nominal $R_{V}=3.1$ will sightly increase the $R_{V}$ values derived for the local M17 dust, but will have little impact on the slope of the relation between $\mathrm{EW}(\mathrm{DIB}) / A_{V}$ and $R_{V}^{-1}$. It could also affect the mean normalised line strengths. The above scenarios and hypotheses can be further tested by examining the relation between EW(DIB)/ $/ A_{V}$ and $R_{V}$ for other star-forming regions that probe a range of extinction properties and grain size distributions, similar to M17. This will also help to disentangle the impact of foreground dust extinction and to determine possible effects due to measurement uncertainties.

\section{Summary}

We present an analysis of the properties of the DIBs in sightlines towards the star-forming region M17 in comparison to the properties of nominal DIBs and of DIBs that have been reported to be anomalous. Our findings can be summarised as follows:

1. Cardelli's extinction law is not suitable for high values of $R_{V}$. The best way of calculating the extinction parameters $A_{V}$ and $E(B-V)$, when the spectral type of the observed star is known, is by directly comparing the observed and intrinsic magnitudes. By using the relation between $R_{V}$ and $E(V-K) / E(B-V)$ of Fitzpatrick \& Massa (2007) it is possible to calculate $A_{K}$ and, therefore the $K$-band excess produced by the circumstellar disk.

2. From the observed $\mathrm{C}_{60}^{+}$DIB at $9577 \AA$ we derive a column density $N\left(\mathrm{C}_{60}^{+}\right)=2.5-4.3 \times 10^{13} \mathrm{~cm}^{-2}$.

3. We also detect the NIR DIBs at 11797,13 176, and $15268 \AA$ in the sightlines towards M17.

4. The profiles of the DIBs towards M17 do not present a red wing in contrast to those towards Herschel 36. This indicates that the red wing is not common to star-forming regions, and that the environment towards Herschel 36 must present some special properties that cause the DIBs to have a red wing.

5. The strength of the $5780 \AA$ DIBs towards M17 is as expected from the relations found in the literature for their values of $E(B-V)$. The $5797 \AA$ DIBs show a relatively large spread in relation to other Galactic DIBs, and the $6614 \AA$ DIBs towards M17 are slightly weaker than expected for their $E(B-V)$ values.

6. In the M17 region we find trends between the strength of the studied DIBs (per unit visual extinction) and $R_{V}^{-1}$, most notably for the 6196 and $7224 \AA$ DIBs. The trend remains when we include the sightlines towards star-forming regions presented in Xiang et al. (2017). For sightlines with $R_{V} \sim 3.1$ there is no trend.

7. The slope values of the linear relation between the DIBs EW normalised by $A_{V}$ and $R_{V}^{-1}$ (DIB- $R_{V}$ sensitivity) allow us to identify two main groups of DIBs in terms of their sensitivity to $R_{V}$ (for $R_{V}>3.8, R_{V}^{-1}<0.26$ ). The first group consists of DIBs with similar sensitivity to changes in $R_{V}$ as the two $C_{60}^{+}$DIBs: 5780, 5797, 7224, 8620, 9577, 9632, and $11797 \AA$. For this group we find that the DIB- $R_{V}$ sensitivity varies linearly with DIB strength. The second group consists of DIBs with roughly three times stronger DIB- $R_{V}$ sensitivity: 4430, 6284, 13176 , and $15268 \AA$; for this group, the DIB- $R_{V}$ sensitivity does not depend on the DIB strength. This response of DIB carriers to changes in interstellar dust properties provides additional clues to the (dis)similarity of different DIB carriers, and may provide clues to their production/destruction mechanisms, and can thus ultimately help with their identifications.

8. Three scenarios are proposed for the observed behaviour of DIBs in star-forming regions (with $R_{V}>3.8$ ): (1) DIB carriers stick to dust grains, and thus get depleted from the gas phase as the grains grow in size (dust-coagulation) in the denser regions of interstellar clouds; (2) DIB carriers are produced from the dust grains in the strong UV radiation fields in these regions which lead to increased abundances of DIB carriers (and that of the $2715 \AA$ band) and, by direct consequence, a decrease in the average dust particle size; (3) as $R_{V}$ decreases and the effective UV radiation field strength increases the ionisation fraction of the parent carriers increases, thus causing the DIBs related to ionised molecular carriers to become stronger. The effective response of DIB strength to changes in the UV radiation field, i.e. $\mathrm{d}\left(\mathrm{EW} / A_{V}\right) / \mathrm{d} R_{V}^{-1}$, is then a measure of the carrier's ionisation potential. At this point we cannot distinguish between these scenarios. Further investigations of specific (star-forming) regions that probe a range of $R_{V}$ values, like M17, are needed to examine the relation between DIB strength and $R_{V}$.

Acknowledgements. Based on observations collected at the European Organisation for Astronomical Research in the Southern Hemisphere under ESO programmes 091.C-0934(B) (Herschel 36), 385.C-0720(A) (HD161056), 60.A-9404(A), 085.D-0741, 089.C-0874(A), and 091.C-0934(B) (M17). The authors thank Rens Waters, Martin Heemskerk, Juan Hernandez Santisteban, Lucia Klarmann, Samayra Straal, Marieke van Doesburgh, Xander Tielens, and Rosine Lallement for discussions that helped to improve this paper. This research made use of Astropy, a community-developed core Python package for Astronomy (Astropy Collaboration 2018), NASA's Astrophysics Data System Bibliographic Services (ADS), and the SIMBAD database, operated at CDS, Strasbourg, France (Wenger et al. 2000).

\section{References}

Astropy Collaboration (Price-Whelan, A. M., et al.) 2018, AJ, 156, 123 Benvenuti, P., \& Porceddu, I. 1989, A\&A, 223, 329

Berné, O., Cox, N. L. J., Mulas, G., \& Joblin, C. 2017, A\&A, 605, L1

Broos, P. S., Feigelson, E. D., Townsley, L. K., et al. 2007, ApJS, 169, 353

Cami, J., \& Cox, N. L. J., eds. 2014, in The Diffuse Interstellar Bands, IAU Symp., 297

Campbell, E. K., Holz, M., Gerlich, D., \& Maier, J. P. 2015, Nature, 523, 322

Capitanio, L., Lallement, R., Vergely, J. L., Elyajouri, M., \& Monreal-Ibero, A. 2017, A\&A, 606, A65

Cardelli, J. A., Clayton, G. C., \& Mathis, J. S. 1989, ApJ, 345, 245

Castelli, F., \& Kurucz, R. L. 2004, ArXiv e-prints [arXiv:astro-ph/0405087]

Cecchi-Pestellini, C., Aiello, S., \& Barsella, B. 1995, ApJS, 100, 187

Chini, R., \& Kruegel, E. 1983, A\&A, 117, 289

Chini, R., Elsaesser, H., \& Neckel, T. 1980, A\&A, 91, 186

Chlewicki, G., van der Zwet, G. P., van Ijzendoorn, L. J., Greenberg, J. M., \& Alvarez, P. P. 1986, ApJ, 305, 455

Churchwell, E. 1990, in Contemporary Mathematics, Vol. 112, Hot Star Workshop III: The Earliest Phases of Massive Star Birth, ed. P. Brown \& W. Fuller (American Mathematical Society) 186

Cox, N. L. J., Kaper, L., Foing, B. H., \& Ehrenfreund, P. 2005, A\&A, 438, 187

Cox, N. L. J., Cordiner, M. A., Ehrenfreund, P., et al. 2007, A\&A, 470, 941

Cox, N. L. J., Cami, J., Kaper, L., et al. 2014, A\&A, 569, A117

Cutri, R. M., Skrutskie, M. F., van Dyk, S., et al. 2003, VizieR Online Data Catalog: II/246

Dahlstrom, J., York, D. G., Welty, D. E., et al. 2013, ApJ, 773, 41

Damineli, A., Almeida, L. A., Blum, R. D., et al. 2016, MNRAS, 463, 2653

de Vries, J., Steger, H., Kamke, B., et al. 1992, Chem. Phys. Lett., 188, 159

Drimmel, R., Cabrera-Lavers, A., \& López-Corredoira, M. 2003, A\&A, 409, 205

Ehrenfreund, P., Cami, J., Jiménez-Vicente, J., et al. 2002, ApJ, 576, L117

Ellerbroek, L. E., Podio, L., Kaper, L., et al. 2013, A\&A, 551, A5

Fitzpatrick, E. L. 1999, PASP, 111, 63

Fitzpatrick, E. L., \& Massa, D. 2007, ApJ, 663, 320 
Freudling, W., Romaniello, M., Bramich, D. M., et al. 2013, A\&A, 559, A96

Friedman, S. D., York, D. G., McCall, B. J., et al. 2011, ApJ, 727, 33

Galazutdinov, G. A., Manicò, G., Pirronello, V., \& Krełowski, J. 2004, MNRAS, 355,169

Geballe, T. R., Najarro, F., Figer, D. F., Schlegelmilch, B. W., \& de La Fuente, D. 2011, Nature, 479,200

Gordon, K. D., \& Clayton, G. C. 1998, ApJ, 500, 816

Gordon, K. D., Clayton, G. C., Misselt, K. A., Landolt, A. U., \& Wolff, M. J. 2003, ApJ, 594, 279

Hamano, S., Kobayashi, N., Kondo, S., et al. 2015, ApJ, 800, 137

Hanson, M. M., Howarth, I. D., \& Conti, P. S. 1997, ApJ, 489, 698

Herbig, G. H. 1993, ApJ, 407, 142

Hobbs, L. M., York, D. G., Thorburn, J. A., et al. 2009, ApJ, 705, 32

Hoffmeister, V. H., Chini, R., Scheyda, C. M., et al. 2008, ApJ, 686, 310

Joblin, C., D'Hendecourt, L., Leger, A., \& Maillard, J. P. 1990, Nature, 346, 729

Jones, A. P. 2004, in Astrophysics of Dust, ed. A. N. Witt, G. C. Clayton, \& B. T. Draine, ASP Conf. Ser., 309, 347

Jura, M. 1980, ApJ, 235, 63

Kausch, W., Noll, S., Smette, A., et al. 2015, A\&A, 576, A78

Kos, J., Zwitter, T., Wyse, R., et al. 2014, Science, 345, 791

Krełowski, J., Ehrenfreund, P., Foing, B. H., et al. 1999, A\&A, 347, 235

Krełowski, J., Galazutdinov, G. A., Bondar, A., \& Beletsky, Y. 2016, MNRAS, 460,2706

Kurucz, R. L. 1993, VizieR Online Data Catalog: VI/039

Maíz Apellániz, J., Barbá, R. H., Sota, A., \& Simón-Díaz, S. 2015, A\&A, 583, A132

Mathis, J. S., Rumpl, W., \& Nordsieck, K. H. 1977, ApJ, 217, 425

McCall, B. J., Drosback, M. M., Thorburn, J. A., et al. 2010, ApJ, 708, 1628

Mennella, V., Colangeli, L., Bussoletti, E., Palumbo, P., \& Rotundi, A. 1998, ApJ, 507, L177

Mishra, A., \& Li, A. 2015, ApJ, 809, 120

Misselt, K. A., Clayton, G. C., \& Gordon, K. D. 1999, ApJ, 515, 128

Modigliani, A., Goldoni, P., Royer, F., et al. 2010, in Observatory Operations: Strategies, Processes, and Systems III, Proc. SPIE, 7737, 773728
Munari, U., Tomasella, L., Fiorucci, M., et al. 2008, A\&A, 488, 969

Oka, T., Welty, D. E., Johnson, S., et al. 2013, ApJ, 773, 42

Ormel, C. W., Paszun, D., Dominik, C., \& Tielens, A. G. G. M. 2009, A\&A, 502, 845

Pecaut, M. J., \& Mamajek, E. E. 2013, ApJS, 208, 9

Povich, M. S., Churchwell, E., Bieging, J. H., et al. 2009, ApJ, 696, 1278 Prisinzano, L., Damiani, F., Micela, G., \& Pillitteri, I. 2007, A\&A, 462, 123

Puls, J., Urbaneja, M. A., Venero, R., et al. 2005, A\&A, 435, 669

Ramírez-Tannus, M. C., Kaper, L., de Koter, A., et al. 2017, A\&A, 604, A78

Rawlings, M. G., Adamson, A. J., \& Whittet, D. C. B. 2003, MNRAS, 341, 1121

Reid, M. J., Menten, K. M., Zheng, X. W., et al. 2009, ApJ, 700, 137

Rivero González, J. G., Puls, J., Najarro, F., \& Brott, I. 2012, A\&A, 537, A79

Ruiterkamp, R., Cox, N. L. J., Spaans, M., et al. 2005, A\&A, 432, 515

Schulz, A., Lenzen, R., Schmidt, T., \& Proetel, K. 1981, A\&A, 95, 94

Smette, A., Sana, H., Noll, S., et al. 2015, A\&A, 576, A77

Snow, T. P. 2014, in The Diffuse Interstellar Bands, eds. J. Cami \& N. L. J. Cox, IAU Symp., 297, 3

Snow, T. P., Welty, D. E., Thorburn, J., et al. 2002, ApJ, 573, 670

Sonnentrucker, P., Cami, J., Ehrenfreund, P., \& Foing, B. H. 1997, A\&A, 327, 1215

Tuairisg, S. Ó., Cami, J., Foing, B. H., Sonnentrucker, P., \& Ehrenfreund, P. 2000, A\&AS, 142, 225

Vernet, J., Dekker, H., D’Odorico, S., et al. 2011, A\&A, 536, A105

Vos, D. A. I., Cox, N. L. J., Kaper, L., Spaans, M., \& Ehrenfreund, P. 2011, A\&A, 533, A129

Wegner, W. 2000, MNRAS, 319, 771

Wegner, W. 2014, Acta Astron., 64, 261

Welty, D. E., Federman, S. R., Gredel, R., Thorburn, J. A., \& Lambert, D. L. 2006, ApJS, 165, 138

Wenger, M., Ochsenbein, F., Egret, D., et al. 2000, A\&AS, 143, 9

Xiang, F. Y., Li, A., \& Zhong, J. X. 2011, ApJ, 733, 91

Xiang, F. Y., Li, A., \& Zhong, J. X. 2017, ApJ, 835, 107

Xu, Y., Moscadelli, L., Reid, M. J., et al. 2011, ApJ, 733, 25

Zasowski, G., \& Ménard, B. 2014, in The Diffuse Interstellar Bands, eds. J. Cami \& N. L. J. Cox, IAU Symp., 297, 68 
Appendix A: Observed DIBs and Gaussian fits
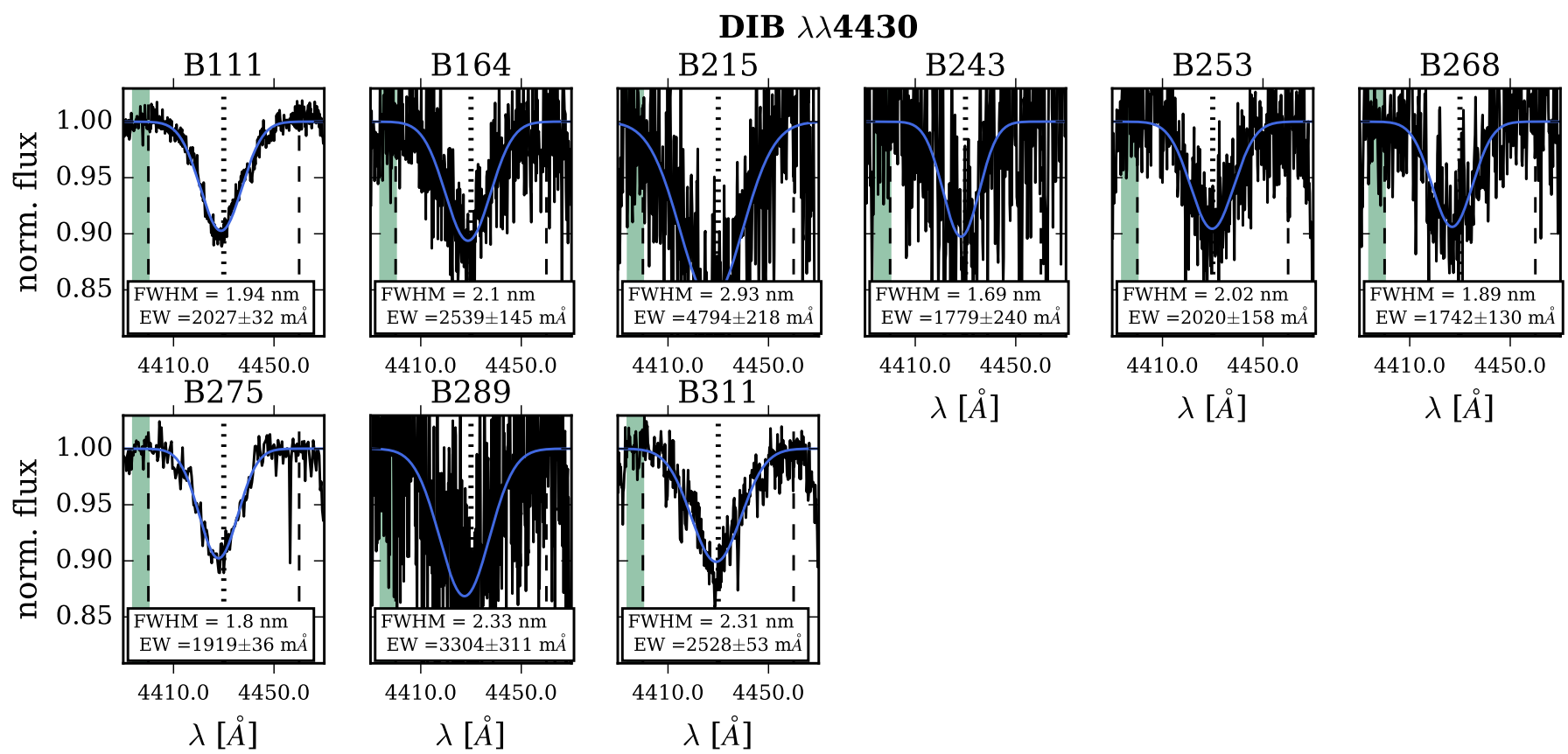

$\lambda[\AA]$

Fig. A.1. DIB profiles at $4430 \AA$. The vertical dashed lines show the integration limits used to calculate the DIB strength, the dotted line shows the central wavelength of the DIB, and the green shaded area shows the region in which the error was calculated. We show the Gaussian fit to the DIB profile with a solid blue line. The white box at the bottom of each panel indicates the FWHM and EW of this DIB for each object.
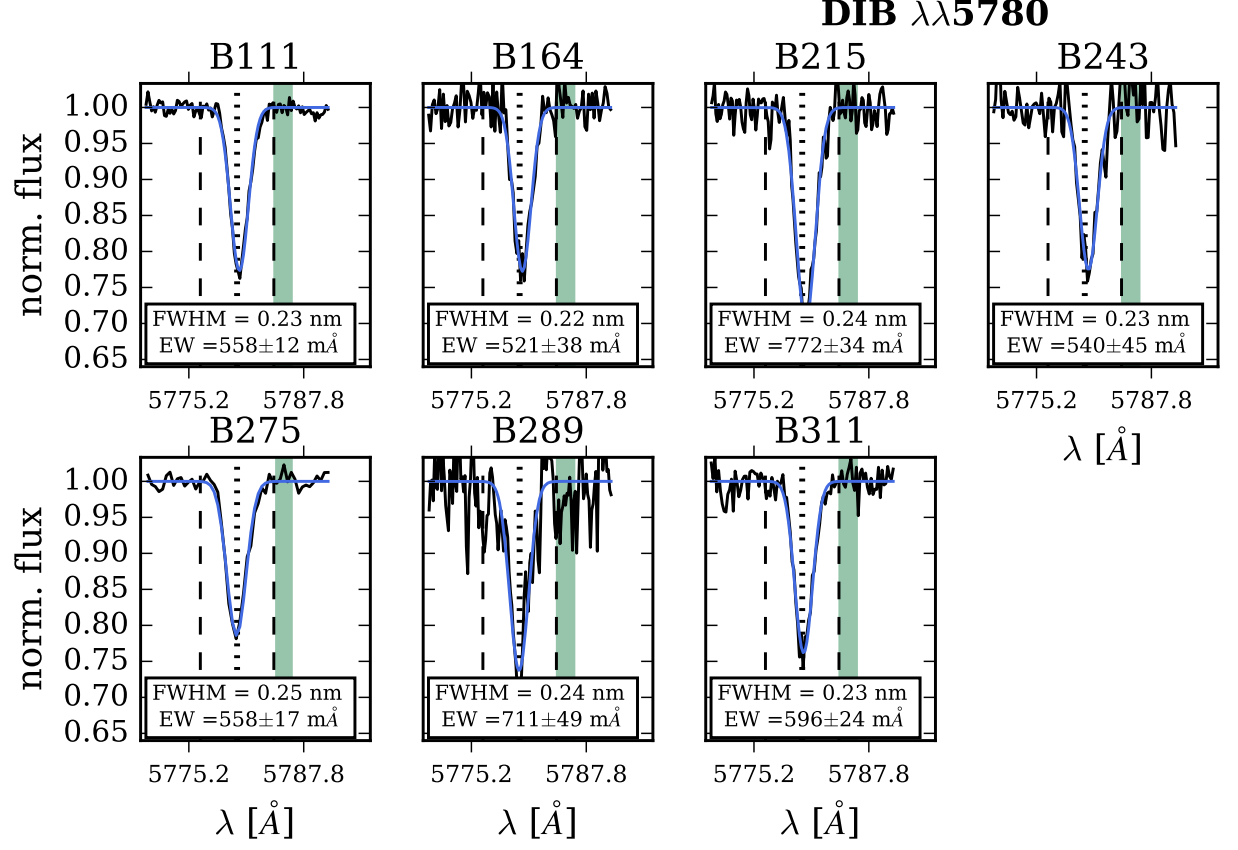

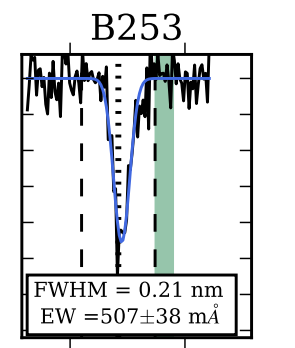

$5775.2 \quad 5787.8$

$\lambda[\AA]$

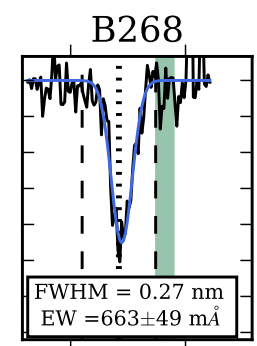

$5775.2 \quad 5787.8$

$\lambda[\AA]$

Fig. A.2. DIB profiles at $5780 \AA$ A. The vertical dashed lines show the integration limits used to calculate the DIB strength, the dotted line shows the central wavelength of the DIB, and the green shaded area shows the region in which the error was calculated. We show the Gaussian fit to the DIB profile with a solid blue line. The white box at the bottom of each panel indicates the FWHM and EW of this DIB for each object. 
DIB $\lambda \lambda \mathbf{5 7 9 7}$
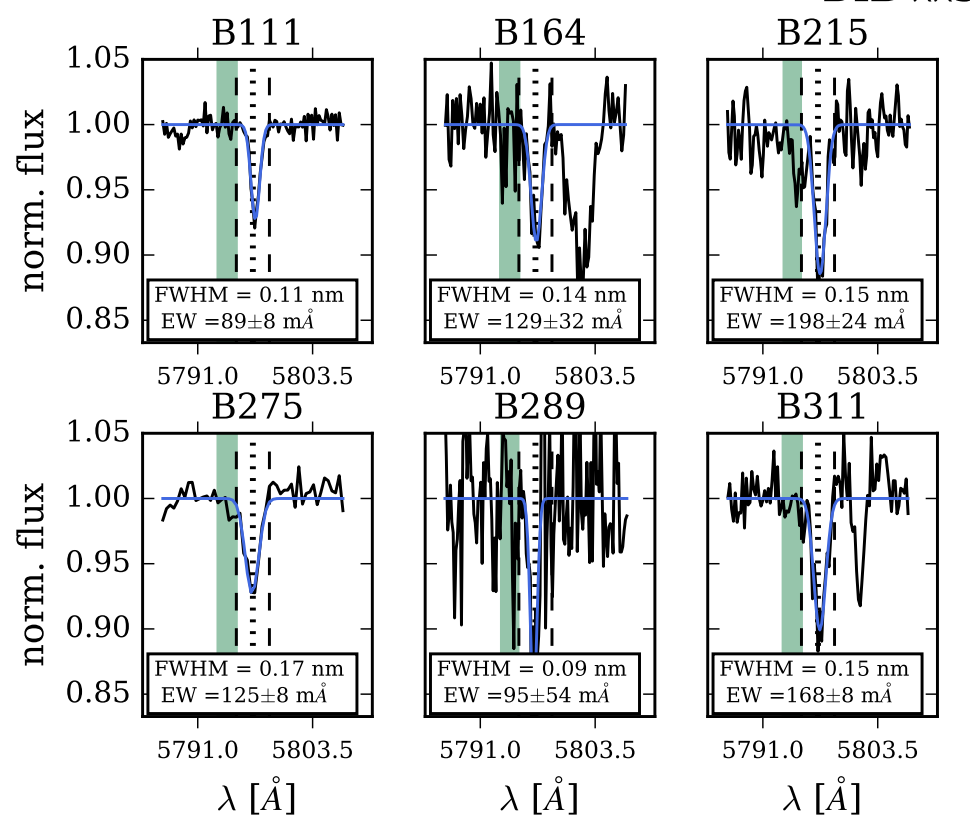

B289

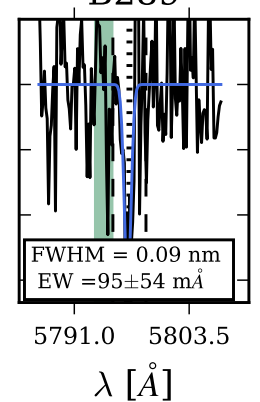

B243

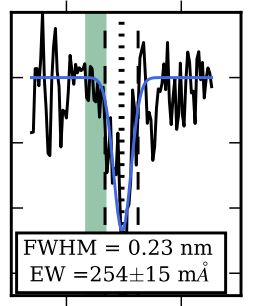

$5791.0 \quad 5803.5$

$\lambda[\AA]$
B253

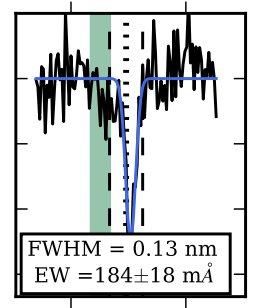

$5791.0 \quad 5803.5$

$\lambda[\AA]$
B268

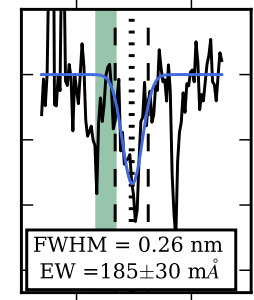

$5791.0 \quad 5803.5$

$\lambda[\AA]$

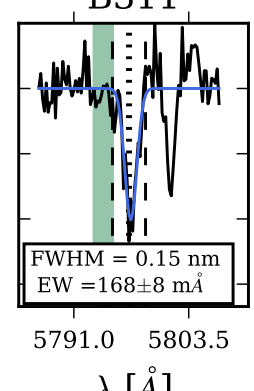

Fig. A.3. DIB profiles at $5798 \AA$. The vertical dashed lines show the integration limits used to calculate the DIB strength, the dotted line shows the central wavelength of the DIB, and the green shaded area shows the region in which the error was calculated. We show the Gaussian fit to the DIB profile with a solid blue line. The white box at the bottom of each panel indicates the FWHM and EW of this DIB for each object.

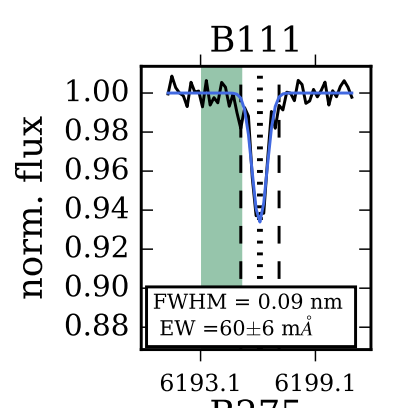

B275

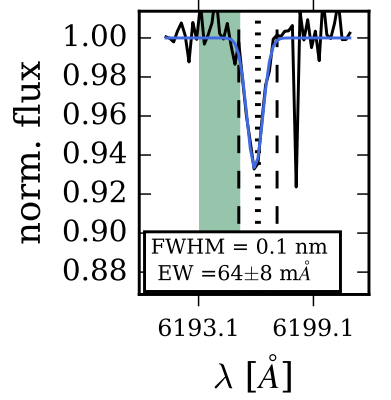

\section{DIB $\lambda \lambda 6196$}

B164

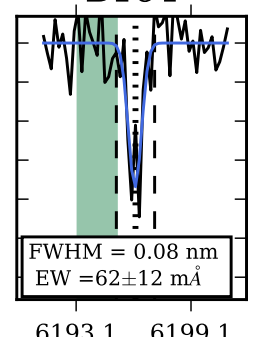

B289

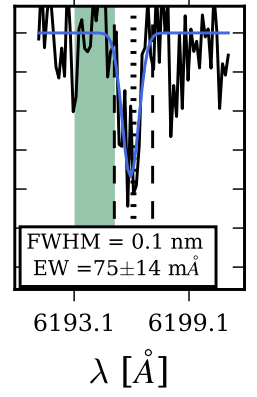

B215

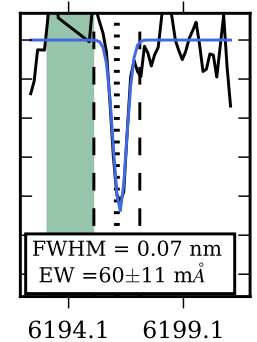

B311

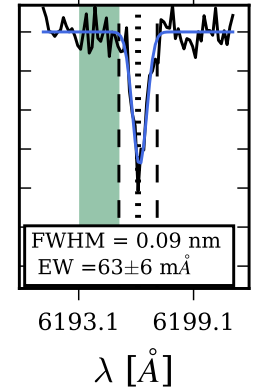

B243

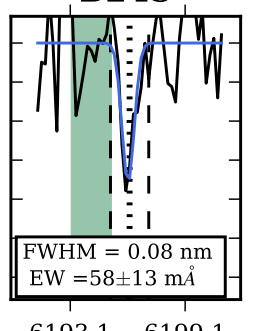

$\lambda[\AA]$
B253

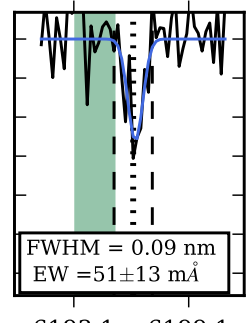

6193.16199 .1

$\lambda[\AA]$
B268

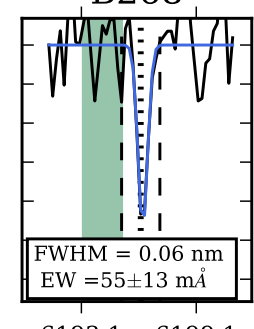

6193.16199 .1

$\lambda[\AA]$

Fig. A.4. DIB profiles at $6196 \AA$ A. The vertical dashed lines show the integration limits used to calculate the DIB strength, the dotted line shows the central wavelength of the DIB, and the green shaded area shows the region in which the error was calculated. We show the Gaussian fit to the DIB profile with a solid blue line. The white box at the bottom of each panel indicates the FWHM and EW of this DIB for each object. 

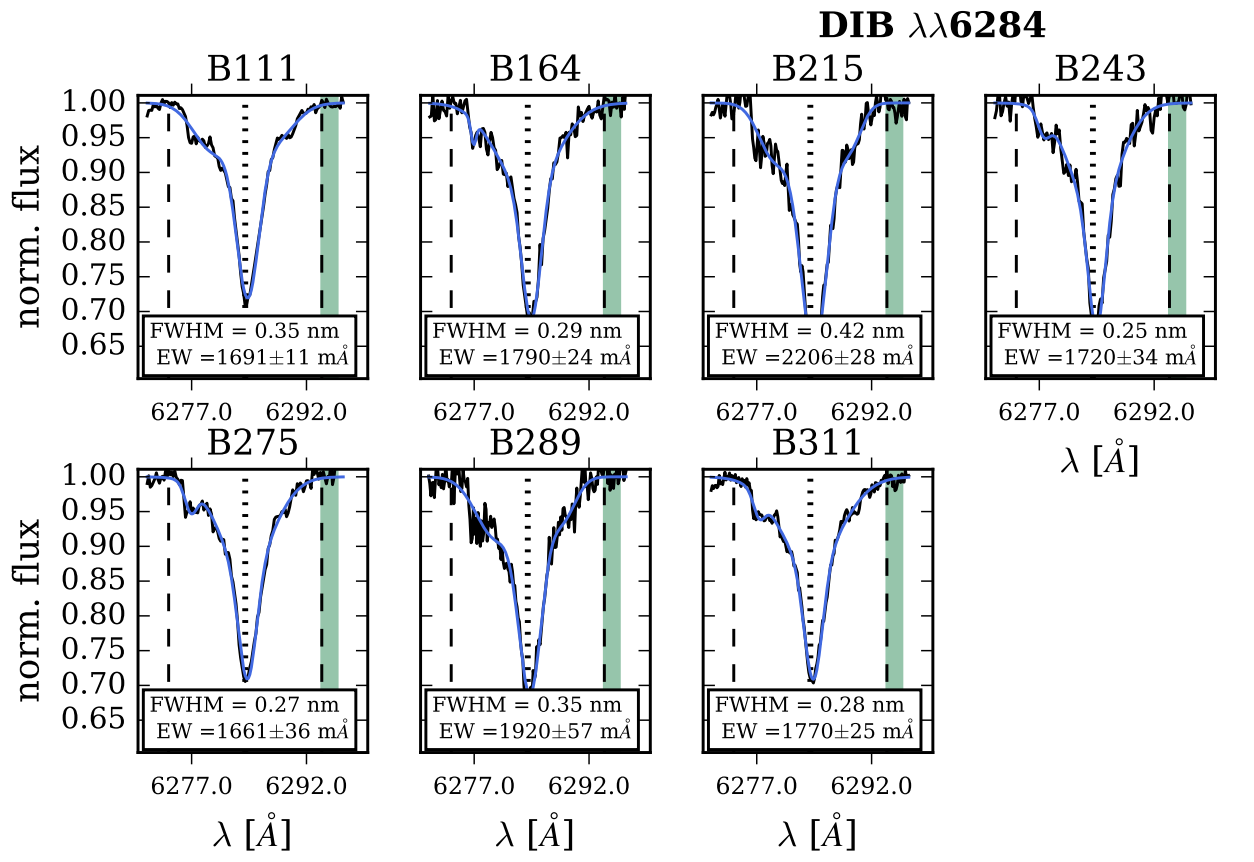

$6277.0 \quad 6292.0$

$\lambda[\AA]$

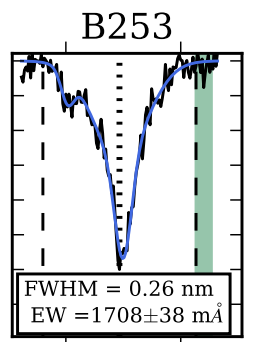

$6277.0 \quad 6292.0$

$\lambda[\AA]$

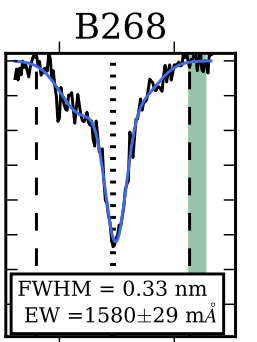

$6277.0 \quad 6292.0$

$\lambda[\AA]$

Fig. A.5. DIB profiles at $6284 \AA$ A. The vertical dashed lines show the integration limits used to calculate the DIB strength, the dotted line shows the central wavelength of the DIB, and the green shaded area shows the region in which the error was calculated. We show the Gaussian fit to the DIB profile with a solid blue line. The white box at the bottom of each panel indicates the FWHM and EW of this DIB for each object.
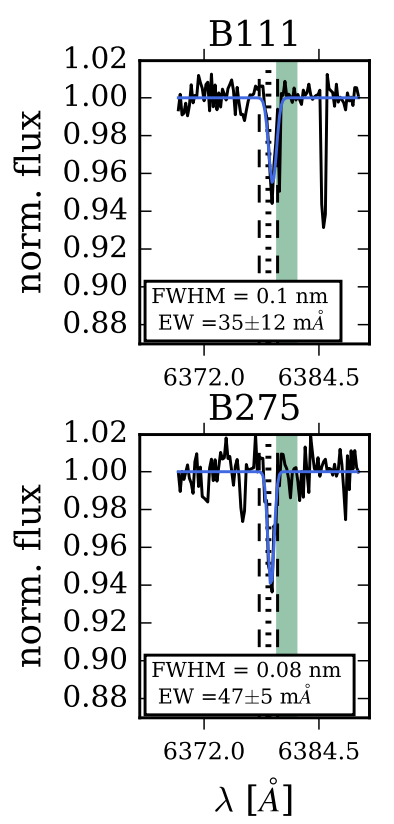

B164

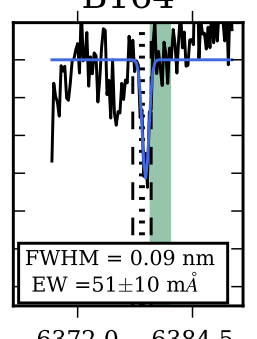

B289

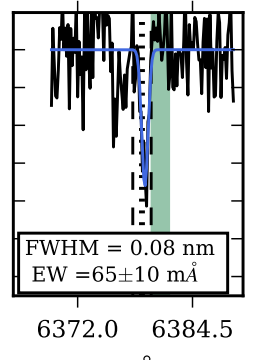

$\lambda[\AA]$
DIB $\lambda \lambda 6379$

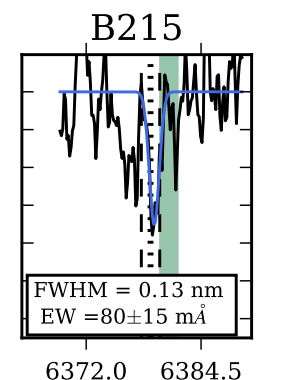

B311

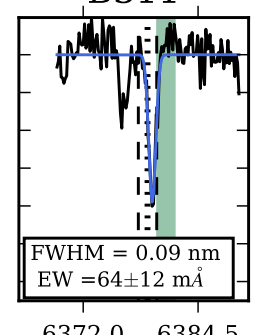

$\lambda[\AA]$

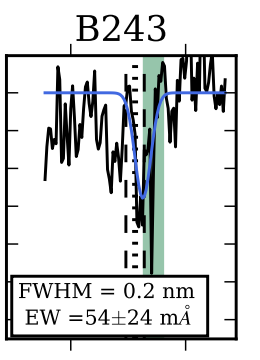

$6372.0 \quad 6384.5$

$\lambda[\AA]$
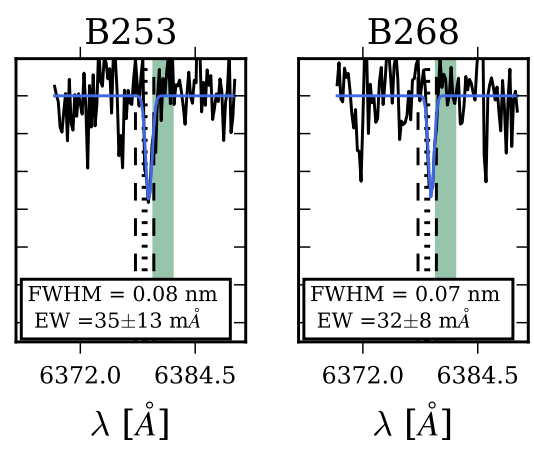

$6372.0 \quad 6384.5$

$\lambda[\AA]$

Fig. A.6. DIB profiles at $6379 \AA$ A. The vertical dashed lines show the integration limits used to calculate the DIB strength, the dotted line shows the central wavelength of the DIB, and the green shaded area shows the region in which the error was calculated. We show the Gaussian fit to the DIB profile with a solid blue line. The white box at the bottom of each panel indicates the FWHM and EW of this DIB for each object. 
DIB $\lambda \lambda 6614$
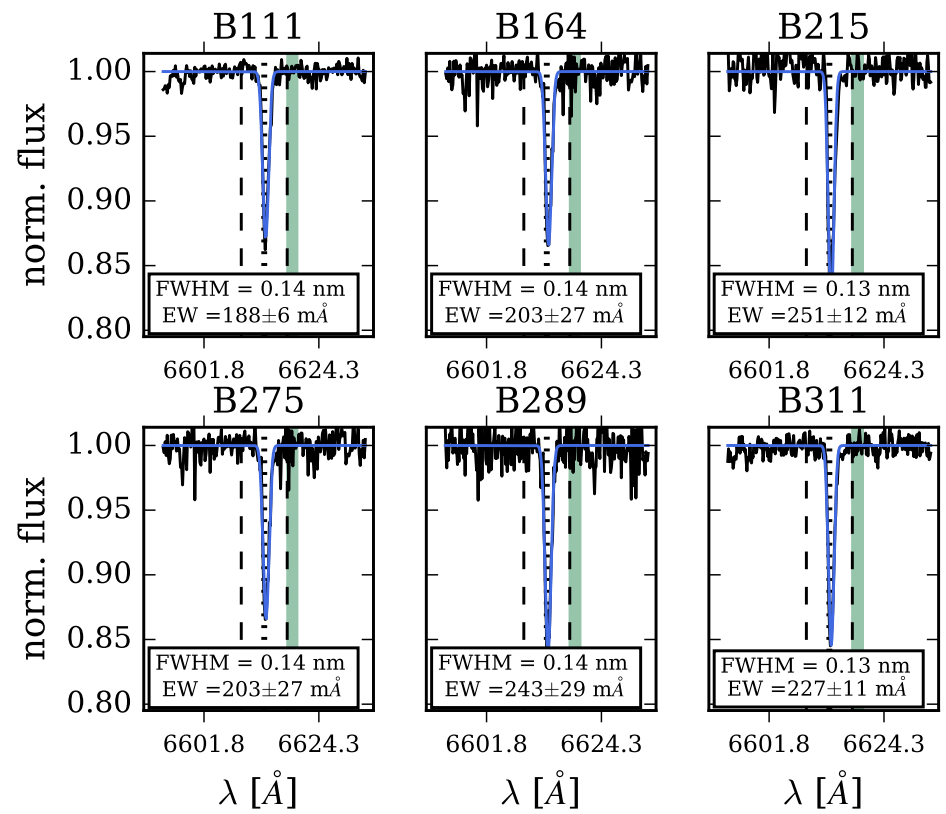

B289

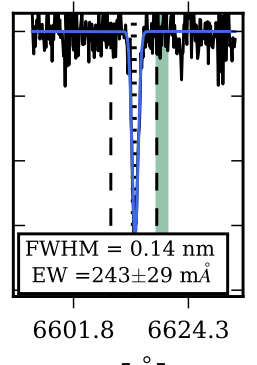

B311

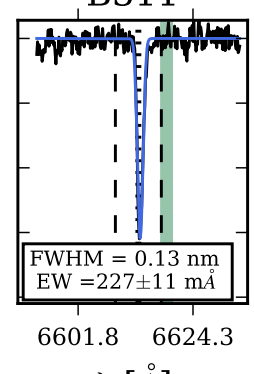

B243

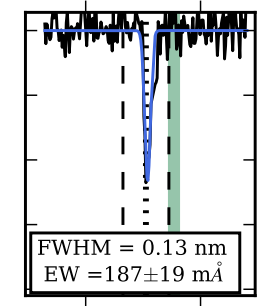

$6601.8 \quad 6624.3$

$\lambda[\AA]$
B253

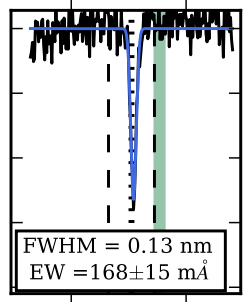

$6601.8 \quad 6624.3$

$\lambda[\AA]$
B268

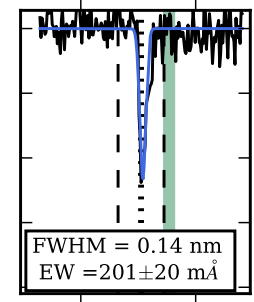

$6601.8 \quad 6624.3$

$\lambda[\AA]$

Fig. A.7. DIB profiles at $6113 \AA$. The vertical dashed lines show the integration limits used to calculate the DIB strength, the dotted line shows the central wavelength of the DIB, and the green shaded area shows the region in which the error was calculated. We show the Gaussian fit to the DIB profile with a solid blue line. The white box at the bottom of each panel indicates the FWHM and EW of this DIB for each object.
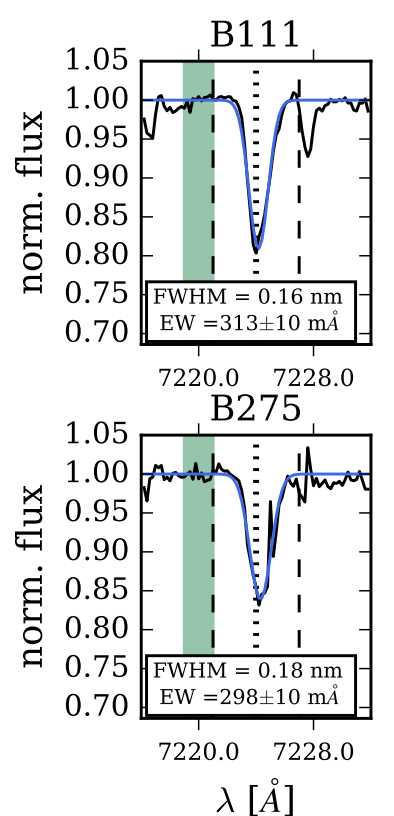

B164

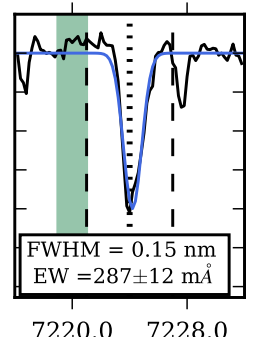

B289

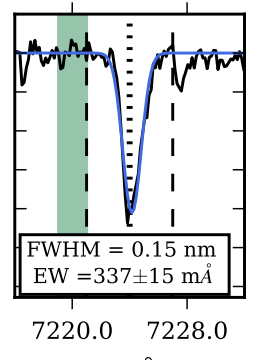

$\lambda[\AA]$
DIB $\lambda \lambda 7224$

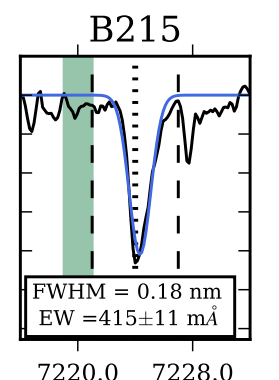

B311

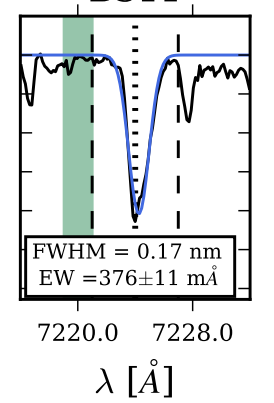

B243

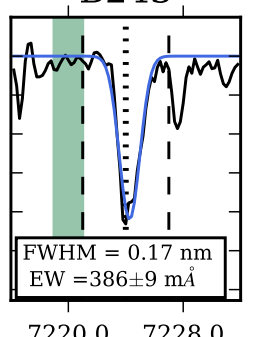

$\lambda[\AA]$

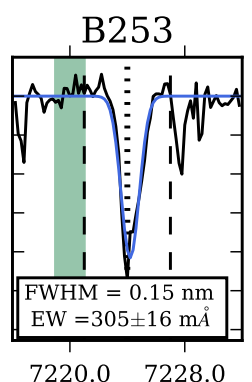

$\lambda[\AA]$
B268

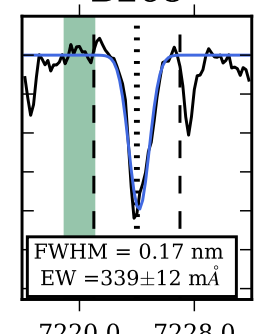

$\lambda[\AA]$

Fig. A.8. DIB profiles at $7224 \AA$. The vertical dashed lines show the integration limits used to calculate the DIB strength, the dotted line shows the central wavelength of the DIB, and the green shaded area shows the region in which the error was calculated. We show the Gaussian fit to the DIB profile with a solid blue line. The white box at the bottom of each panel indicates the FWHM and EW of this DIB for each object. 

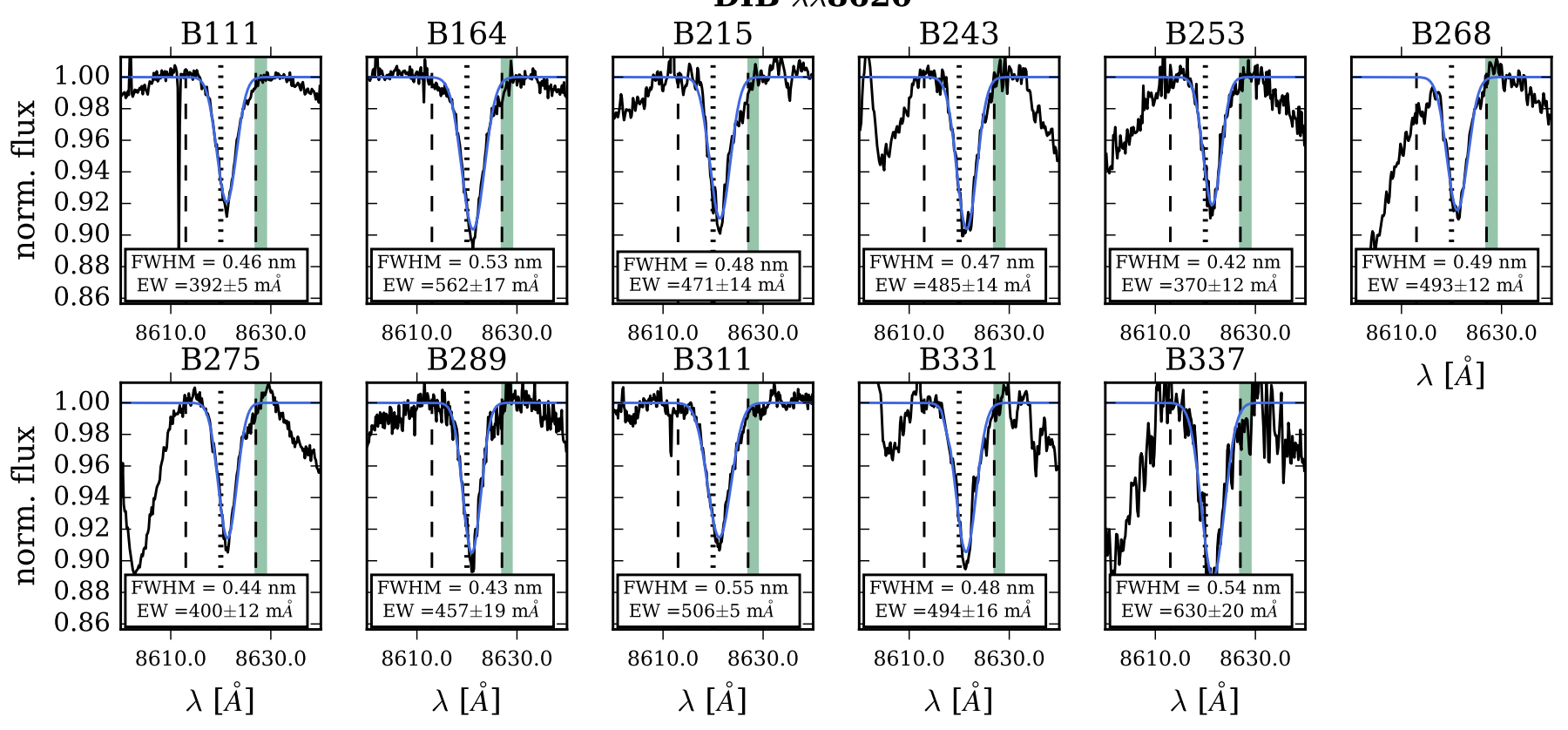

$8610.0 \quad 8630.0$

$\lambda[\AA]$

Fig. A.9. DIB profiles at $8620 \AA$. The vertical dashed lines show the integration limits used to calculate the DIB strength, the dotted line shows the central wavelength of the DIB, and the green shaded area shows the region in which the error was calculated. We show the Gaussian fit to the DIB profile with a solid blue line. The white box at the bottom of each panel indicates the FWHM and EW of this DIB for each object.
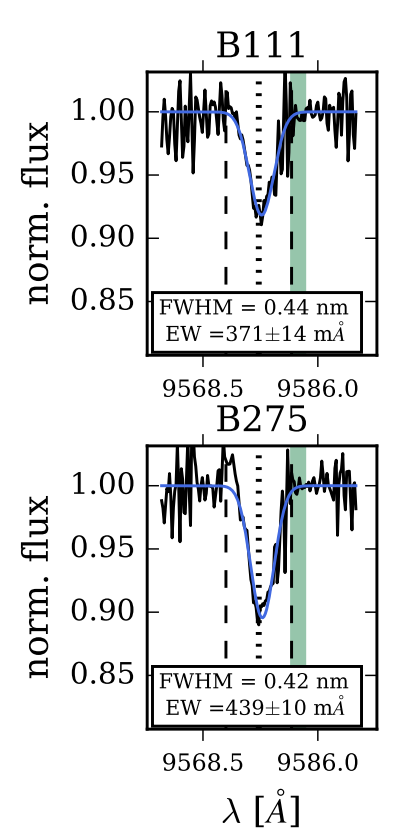

$\mathrm{C}_{60}^{+} \lambda \lambda \mathbf{9 5 7 7}$
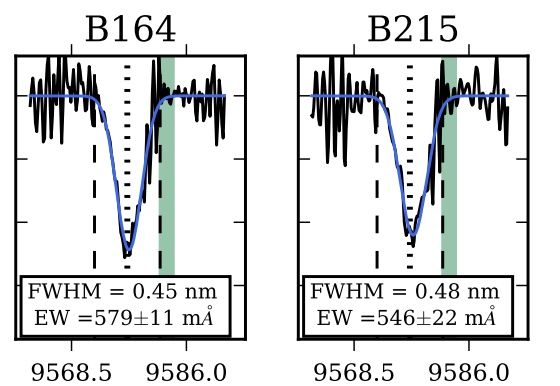

B311

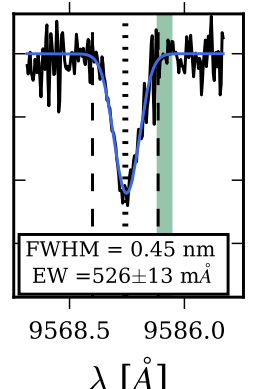

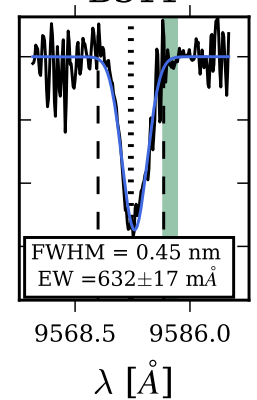

B243

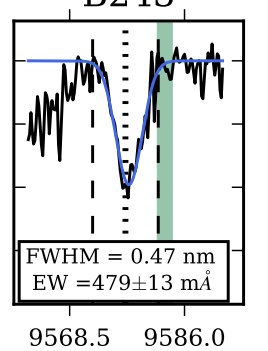

B331

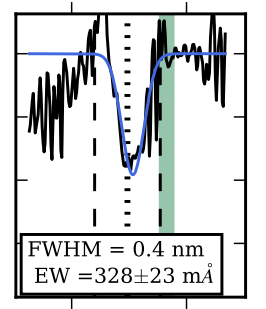

9568.59586 .0

$\lambda[\AA]$

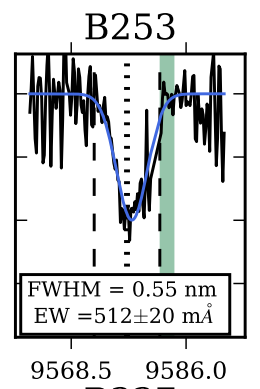

B337

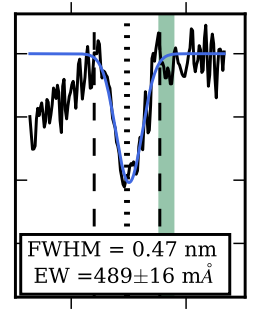

$9568.5 \quad 9586.0$

$\lambda[\AA]$

Fig. A.10. $C_{60}^{+}$DIB profiles at $9577 \AA$. The vertical dashed lines show the integration limits used to calculate the DIB strength, the dotted line shows the central wavelength of the DIB, and the green shaded area shows the region in which the error was calculated. We show the Gaussian fit to the DIB profile with a solid blue line. The white box at the bottom of each panel indicates the FWHM and EW of this DIB for each object. 
$\mathrm{C}_{60}^{+} \lambda \lambda \mathbf{9 6 3 2}$

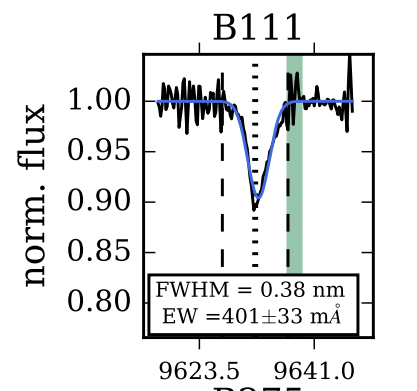

B275

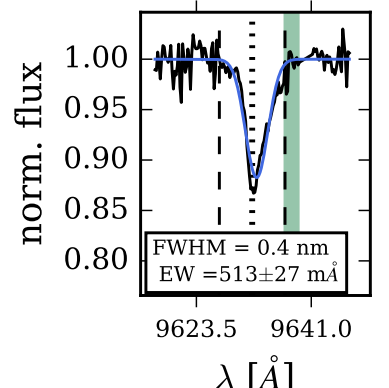

B164

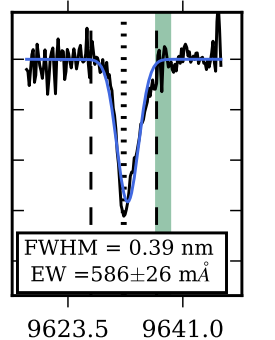

B289

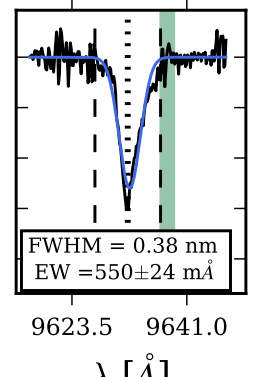

B215

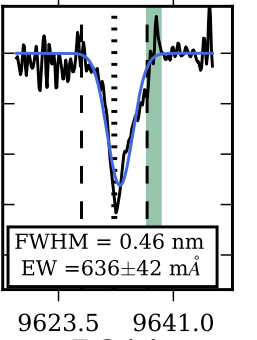

B311

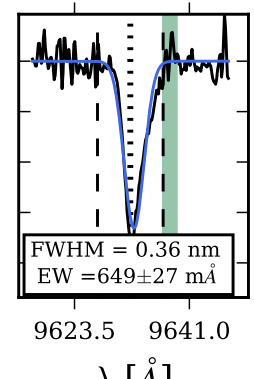

B243

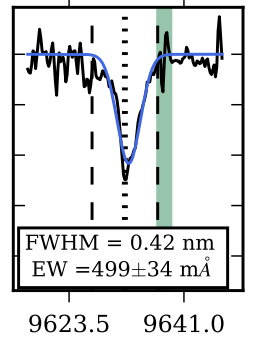

B331

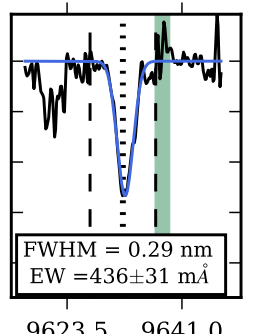

$\lambda[\AA]$

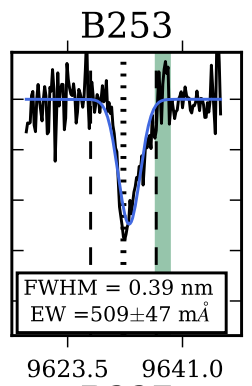

B337

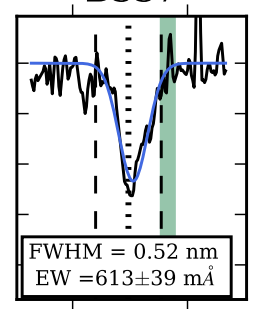

9623.59641 .0

$\lambda[\AA]$

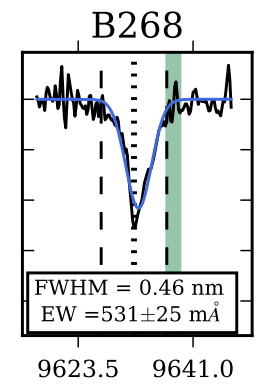

$\lambda[\AA]$

Fig. A.11. $C_{60}^{+}$DIB profiles at $9632 \AA$. The vertical dashed lines show the integration limits used to calculate the DIB strength, the dotted line shows the central wavelength of the DIB, and the green shaded area shows the region in which the error was calculated. We show the Gaussian fit to the DIB profile with a solid blue line. The white box at the bottom of each panel indicates the FWHM and EW of this DIB for each object.

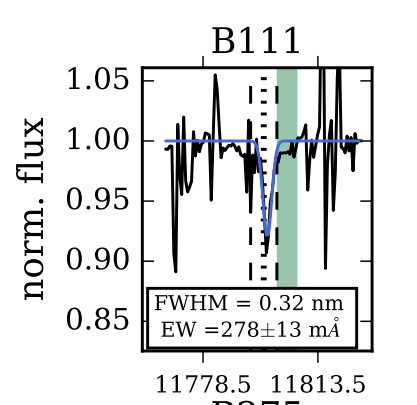

B275

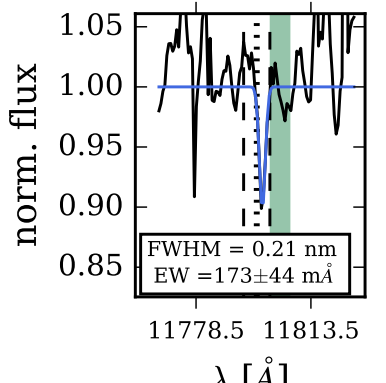

B164

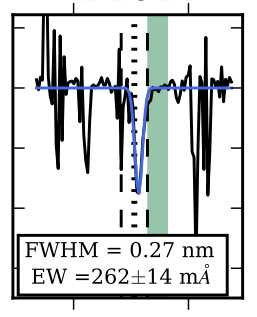

11778.511813 .5 B289

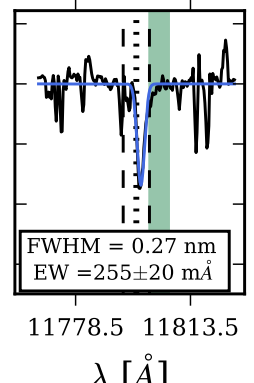

DIB $\lambda \lambda 11797$

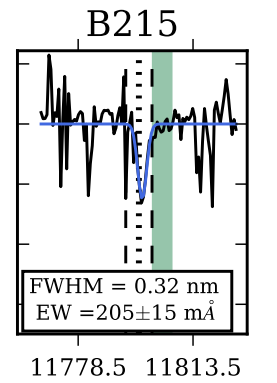

B311

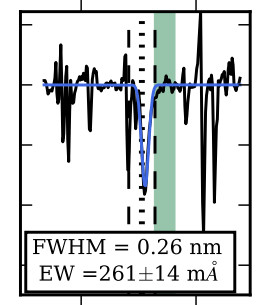

11778.511813 .5

$\lambda[\AA]$

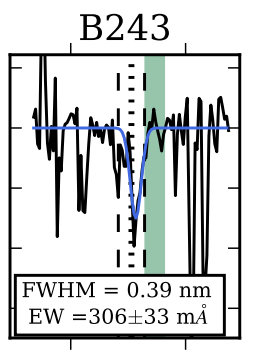

11778.511813 .5 B331

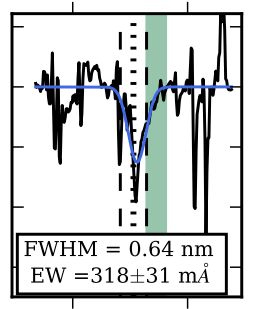

11778.511813 .5

$\lambda[\AA]$

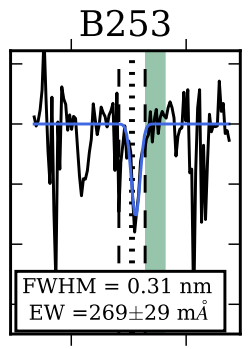

11778.511813 .5 B337

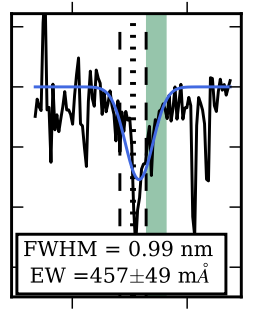

11778.511813 .5

$\lambda[\AA]$

Fig. A.12. NIR DIB profiles at $11797 \AA$. The vertical dashed lines show the integration limits used to calculate the DIB strength, the dotted line shows the central wavelength of the DIB, and the green shaded area shows the region in which the error was calculated. We show the Gaussian fit to the DIB profile with a solid blue line. The white box at the bottom of each panel indicates the FWHM and EW of this DIB for each object. 

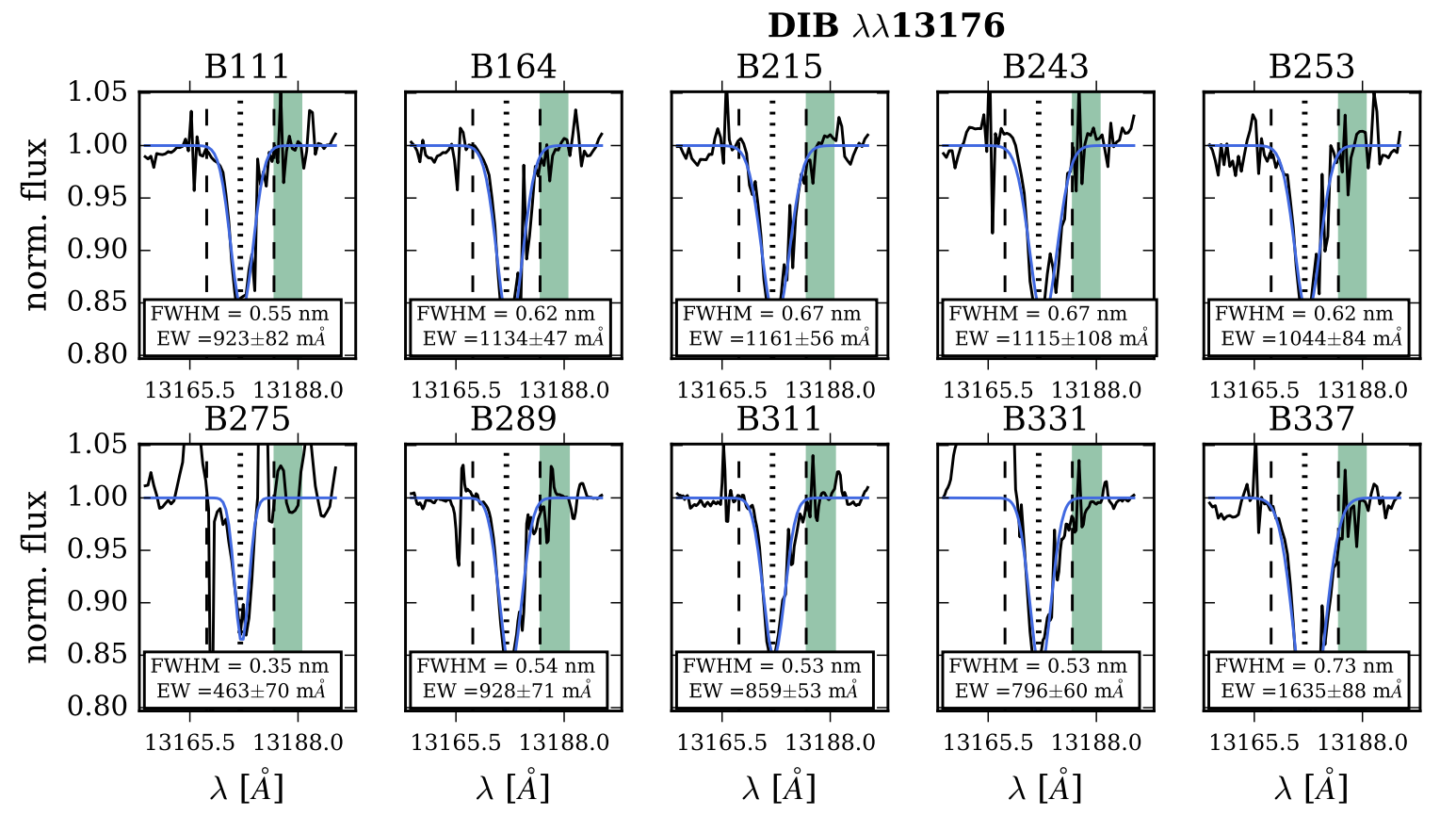

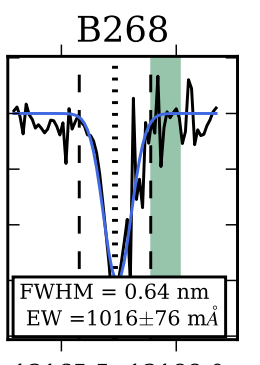

13165.513188 .0

$\lambda[\AA]$

Fig. A.13. NIR DIB profiles at $13176 \AA$. The vertical dashed lines show the integration limits used to calculate the DIB strength, the dotted line shows the central wavelength of the DIB, and the green shaded area shows the region in which the error was calculated. We show the Gaussian fit to the DIB profile with a solid blue line. The white box at the bottom of each panel indicates the FWHM and EW of this DIB for each object.
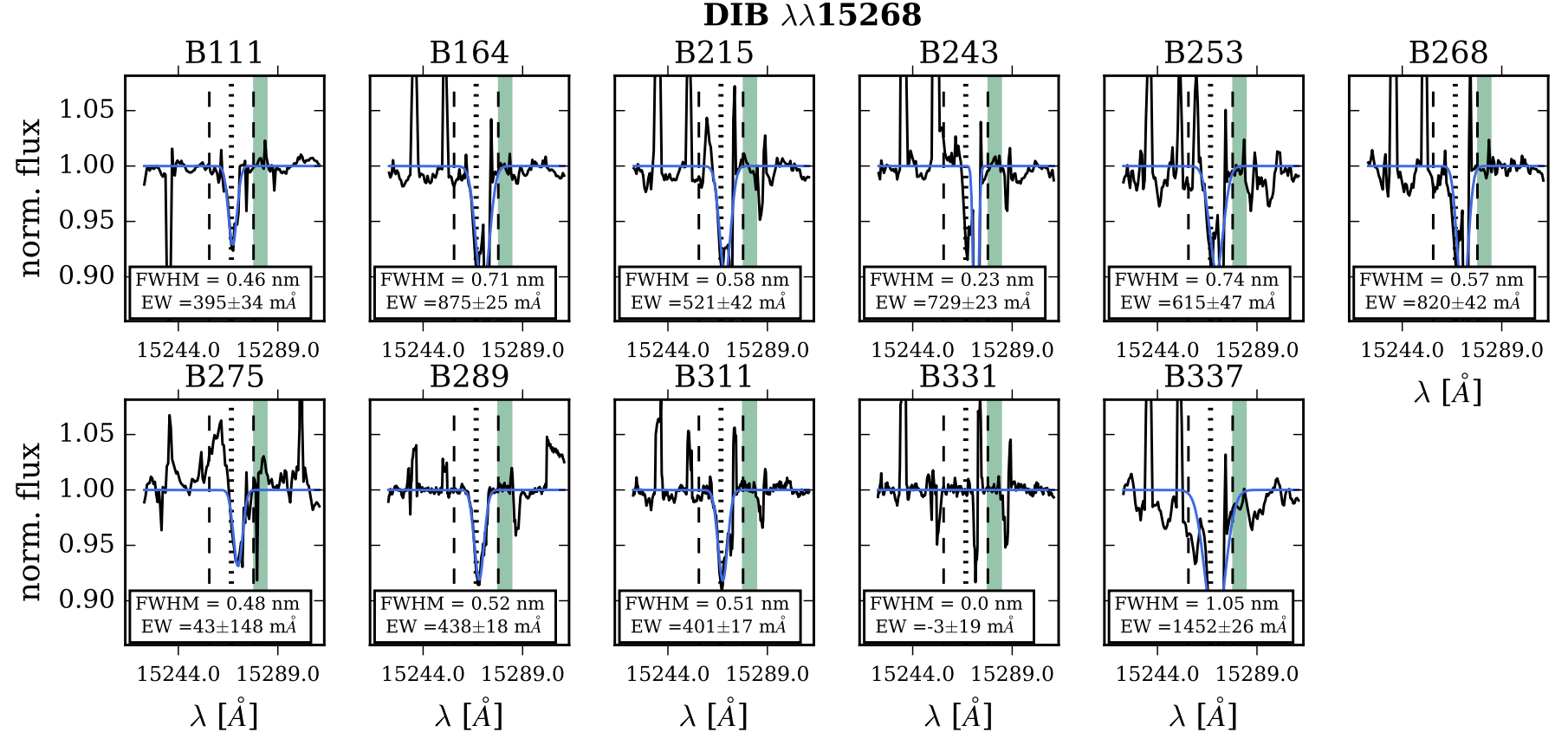

Fig. A.14. NIR DIB profiles at $15268 \AA$. The vertical dashed lines show the integration limits used to calculate the DIB strength, the dotted line shows the central wavelength of the DIB, and the green shaded area shows the region in which the error was calculated. We show the Gaussian fit to the DIB profile with a solid blue line. The white box at the bottom of each panel indicates the FWHM and EW of this DIB for each object. 